\title{
Bounds for the degrees of CM-fields of class number one
}

\author{
by \\ Sofiène Bessassi (Caen)
}

1. Introduction. A number field $K$ is said to be a $C M$-field if $K$ is a totally imaginary quadratic extension of its maximal totally real subfield $k$. According to class field theory, if $K$ is a CM-field then the class number $h_{k}$ of $k$ divides the class number $h_{K}$ of $K$ and $h_{K}^{-}=h_{K} / h_{k}$, which is a divisor of $h_{K}$, is called the relative class number of $K$. If $n$ denotes the degree of $k$, then $K$ is of even degree $2 n$. Notice that an imaginary abelian number field is always a CM-field, whereas a normal imaginary number field is a CM-field if and only if the complex conjugation is in the center of its Galois group $\operatorname{Gal}(K / \mathbb{Q})$ (which implies that $k$ also is a normal number field).

In 1974, A. M. Odlyzko proved that there are only finitely many normal CM-fields of a given class number (see [22]). In 1979, J. Hoffstein showed that normal CM-fields of degrees greater than or equal to 436 have relative class number greater than one (see [11]). In 1994, K. Yamamura completed the determination of all the abelian CM-fields of class number one: there are 172 such CM-fields and their degrees are less than or equal to 24 (see [36]). Since 1994, various authors have been working on the determination of all the non-abelian normal CM-fields with class number one (see [5], [12]-[14], [15], [16], [20], [26]). The current strategy for solving this problem is first to solve this class number one problem for the normal CM-fields of a given Galois group (see [12], [13], [16], [20]), namely the normal CM-fields of any degree with dihedral or dicyclic Galois group (the reason why these two situations are of paramount importance is that if $K$ is a non-abelian normal CM-field of degree $4 p, p$ any odd prime, then since the complex conjugation must be in the center of its Galois group, this Galois group is isomorphic either to the dihedral group of order $4 p$ or to the dicyclic group of order $4 p$ ). Then one tries to solve the class number one problem for the non-abelian

2000 Mathematics Subject Classification: Primary 11R42, 11R29; Secondary 11G15.

Key words and phrases: CM-fields, relative class numbers, Dedekind zeta functions, Weil's explicit formula. 
normal CM-fields of a given reasonable degree $2 n$ (see [5], [14], [15], [26]). Up to now, this strategy has made it possible to determine all the non-abelian normal CM-fields of degree $2 n \leq 48$. In particular, in this process examples have been found of non-abelian normal CM-fields with class number one of degrees 8, 12, 16, 20, 24, 32, 36, 40 and 48. The following theorem gives the CM-field of class number one and of highest degree known to date:

Theorem 1 (K.-Y. Chang and S.-H. Kwon, see [5]). Let $K_{12}$ be the dihedral field of degree 12 cyclic over $\mathbb{Q}(\sqrt{101})$, namely $K_{12}=\mathbb{Q}(\sqrt{5}, \sqrt{101}, \theta)$ with $\theta^{3}-\theta^{2}-5 \theta-1=0$. The narrow class group of $K_{12}$ is cyclic of order 4. Let $N$ be the Hilbert class field in the narrow sense of $K_{12}$. Then $N$ is a normal CM-field of degree 48 and of class number one. Moreover, $D_{N}=D_{K_{12}}^{4}=2^{32} \cdot 5^{24} \cdot 101^{24}$ and $\varrho_{N}=D_{N}^{1 / 48}=2^{2 / 3} \cdot 5^{1 / 2} \cdot 101^{1 / 2}=35.67 \ldots$

In order to make this strategy more reasonable it would be rather useful to have beforehand a bound for the degrees $2 n$ of the non-abelian normal CM-fields with class number one which would be much smaller than J. Hoffstein's bound $2 n \leq 434$. The aim of this paper is to prove that normal CM-fields with class number one are of degree $2 n \leq 266$. Moreover, we will also prove that, assuming the Generalized Riemann Hypothesis, normal CM-fields with class number one are of degree $2 n \leq 164$, and that CM-fields with class number one are of degree $2 n \leq 174$. Not only will we improve upon J. Hoffstein's bound for the degree of the normal CM-fields with class number one, but we will also improve upon J. Hoffstein's bounds for the root discriminants of the normal CM-fields of a given degree with class number one. We emphasize that we will also prove that the Dedekind zeta function of an imaginary quadratic field $F$ of absolute discriminant $D_{F}$ has no real zero in the range $1-6 /\left(\pi \sqrt{D_{F}}\right) \leq s<1$.

If $D_{k}$ denotes the absolute value of the discriminant of a number field $k$ of degree $n \geq 1$, then its root discriminant $\varrho_{k}$ is defined as $\varrho_{k}=D_{k}^{1 / n}$. We will prove

Theorem 2. Let $K$ be a $C M$-field of degree $2 n$ and $k$ its maximal totally real subfield ( $k$ is of degree $n$ ).

1. Assume the Generalized Riemann Hypothesis and $K$ normal. Then $h_{K}^{-}>1$ if $n \geq 83$, or if $n \leq 82$ but the root discriminant $\varrho_{K}=D_{K}^{1 /(2 n)}$ is larger than the following conditional bounds:

Table 1. Conditional bounds, normal case

\begin{tabular}{ccccccc}
\hline$n$ & 82 & 70 & 60 & 50 & 40 & 30 \\
$D_{K}^{1 /(2 n)}$ & 54.76 & 65.76 & 81.93 & 114.5 & 204.0 & 729.3 \\
\hline$n$ & 24 & 23 & 20 & 15 & 10 & 5 \\
$D_{K}^{1 /(2 n)}$ & 5252 & 6354 & 6499 & 6875 & 7653 & 10250 \\
\hline
\end{tabular}


2. Do not assume the Generalized Riemann Hypothesis anymore, but assume $K$ normal. Then $h_{K}^{-}>1$ if $n \geq 134$, or if $n \leq 133$ but the root discriminant $\varrho_{K}=D_{K}^{1 /(2 n)}$ is larger than the following conditional bounds:

Table 2. Unconditional bounds, normal case

\begin{tabular}{ccc|ccc}
\hline$n$ & $D_{K}^{1 /(2 n)}$ & J. Hoffstein's bounds & $n$ & $D_{K}^{1 /(2 n)}$ & J. Hoffstein's bounds \\
\hline 133 & 44.83 & & 50 & 195.5 & 879 \\
130 & 45.66 & & 40 & 422.3 & \\
120 & 48.84 & \multirow{2}{*}{115} & 30 & 2379 & \\
110 & 52.91 & & 27 & 6783 & \\
100 & 58.30 & \multirow{2}{*}{254} & 26 & 8355 & \\
90 & 65.76 & & 20 & 9207 & \\
80 & 76.71 & \multirow{2}{*}{239} & 15 & 10470 & \\
70 & 94.18 & & 10 & 13100 & \\
60 & 125.8 & \multirow{2}{*}{494} & 5 & 22090 & \\
\hline
\end{tabular}

3. Assume the Generalized Riemann Hypothesis, but do not assume K normal. Then $h_{K}^{-}>1$ if $n \geq 88$, or if $n \leq 87$ but the root discriminant $\varrho_{K}=D_{K}^{1 /(2 n)}$ is larger than the following conditional bounds:

Table 3. Conditional bounds, non-normal case

\begin{tabular}{cccccccc}
\hline$n$ & 87 & 80 & 70 & 60 & 50 & 40 & 30 \\
$D_{K}^{1 /(2 n)}$ & 56.56 & 62.12 & 73.74 & 93.81 & 135.4 & 255.6 & 1052 \\
\hline$n$ & 25 & 24 & 20 & 15 & 10 & 5 & \\
$D_{K}^{1 /(2 n)}$ & 5627 & 6973 & 7258 & 7823 & 8951 & 12460 & \\
\hline
\end{tabular}

Remarks. 1. Without assuming the Generalized Riemann Hypothesis, it is not known how to obtain lower bounds for $h_{K}^{-}$increasing to infinity with $n$ in the non-normal case.

2. We have $\varrho_{K}=D_{K}^{1 /(2 n)} \geq D_{k}^{1 / n}=\varrho_{k}$. Since the lower bounds of discriminants for the totally real fields are much better than for the totally imaginary fields, we always use the former. We denote by $\varrho_{n}$ A. M. Odlyzko's conditional lower bounds for the root discriminants of the totally real number fields of degrees $\geq n$ (under the Generalized Riemann Hypothesis), and by $\varrho_{n}^{\prime}$ L. Tartar's unconditional lower bounds for the root discriminants of the totally real number fields of degrees $\geq n$ (without assuming the Generalized Riemann Hypothesis). We will use the values $\varrho_{n}=54.8874$ for $n=83$, $\varrho_{n}=56.2325$ for $n=88$, and $\varrho_{n}^{\prime}=44.6377$ for $n=134$.

2. Outline of our method. Let $K$ be a CM-field of degree $2 n$, and $k$ its maximal totally real subfield. The starting point is a lower bound for $h_{K}^{-}$. Let $D_{K}$ and $D_{k}$ denote the absolute values of the discriminants of $K$ 
and $k$, and $\operatorname{Res} \zeta_{K}$ and $\operatorname{Res} \zeta_{k}$ the residues at $s=1$ of the Dedekind zeta functions of $K$ and $k$. Let $W_{K}$ be the group of complex roots of unity in $K$, let $w_{K} \geq 2$ be the order of this group, let $E_{K}$ be the unit group of $K$, and let $E_{k}$ be the unit group of $k$. Let $Q_{K}=\left[E_{K}: W_{K} E_{k}\right]$ be the Hasse unit index of $K$. We have $Q_{K} \in\{1,2\}$ (see for example [35, Theorem 4.12]).

The analytic class number formula for $k$ and $K$ yields

$$
h_{K}^{-}=\frac{Q_{K} w_{K}}{(2 \pi)^{n}} \sqrt{\frac{D_{K}}{D_{k}}} \cdot \frac{\operatorname{Res} \zeta_{K}}{\operatorname{Res} \zeta_{k}} .
$$

Thus, to obtain lower bounds for $h_{K}^{-}$which go to infinity with $n$, we will need lower bounds for $D_{K} / D_{k}$, upper bounds for $\operatorname{Res} \zeta_{k}$ and lower bounds for $\operatorname{Res} \zeta_{K}$. For all the three problems, we propose improvements over J. Hoffstein's methods. For both the discriminants and residues, we will make use of one of A. Weil's explicit formulas, which for a number field $E$ of degree $n=r_{1}+2 r_{2}$ reads

$$
\begin{aligned}
\log D_{E}= & n \log \left(8 \pi e^{\gamma}\right)+r_{1} \frac{\pi}{2}-n I_{n, r_{1}}(F)+\sum_{\varrho} \Phi(\varrho) \\
& +2 \sum_{\mathfrak{p}, m} \frac{\log \mathrm{N}(\mathfrak{p})}{(\mathrm{N}(\mathfrak{p}))^{m / 2}} F(m \log \mathrm{N}(\mathfrak{p}))
\end{aligned}
$$

where $F$ is a real-valued even function that must be chosen subject to certain conditions (see Proposition 3 below), the first sum is over the non-trivial zeros $\varrho$ of the Dedekind zeta function of $E$ (those of real part $\beta=\operatorname{Re} \varrho$ with $0<\beta<1$ ), the second double sum is over the prime ideals $\mathfrak{p}$ of $E$,

$$
\Phi(s)=\int_{-\infty}^{\infty} F(x) e^{(s-1 / 2) x} d x
$$

is the Mellin transform of $F$ and

$$
\begin{aligned}
I_{n, r_{1}}(F)= & \int_{0}^{\infty} \frac{1-F(x)}{2 \sinh (x / 2)} d x+\frac{r_{1}}{n} \int_{0}^{\infty} \frac{1-F(x)}{2 \cosh (x / 2)} d x \\
& +\frac{4}{n} \int_{0}^{\infty} F(x) \cosh (x / 2) d x
\end{aligned}
$$

(see for example Poitou [28] for a development of this formula). Notice that for a totally real field, $n=r_{1}$ and

$$
I_{n, n}(F)=\frac{4}{n} \int_{0}^{\infty} F(x) \cosh (x / 2) d x+\int_{0}^{\infty} \frac{(1-F(x)) e^{x / 2}}{\sinh (x)} d x .
$$

If we limit ourselves to functions $F$ for which the last two terms in A. Weil's formula (2) are non-negative, then we obtain lower bounds for discriminants (of number fields of degree $n$ ) which go to infinity with $n$. 
It remains to choose the best possible $F$ to get the best possible bound. We do not try to improve on the known results on the subject. J.-P. Serre, A. M. Odlyzko, G. Poitou, B. Perrin-Riou and L. Tartar have found very good functions $F$ that suit our purposes. Using their choices, we already have a tremendous improvement over the bounds that J. Hoffstein was working with. We will briefly recall those results.

We will also use A. Weil's formula to get an upper bound for the residues at $s=1$ of Dedekind zeta functions of number fields. This approach is new and more complex, we do not ignore the last term in A. Weil's formula and choose $F$ accordingly. This will be detailed in Section 3.

Various ideas can be put together to further improve on J. Hoffstein's lower bounds for residues. This will be dealt with in Sections 4-6.

As a conclusion, we present the explicit bounds in Section 7.

\section{Upper bounds for residues of zeta functions of totally real number fields}

3.1. Lower bounds for discriminants of number fields. We briefly recall the methods for obtaining the currently best known lower bounds for discriminants, as we will need them to estimate residues at $s=1$ of Dedekind zeta functions of number fields. In the following, the notations $n, D_{k}, \mathfrak{p}, \varrho$ are associated to a totally real number field $k$. In particular, $\varrho$ designates the non-trivial zeros of the Dedekind zeta function $\zeta_{k}$ of $k$, i.e. those with $0<\operatorname{Re} \varrho<1$.

All we ask of $F$ is that it is even, of bounded variation and that the sums we write make sense. Furthermore, for the purpose of getting lower bounds for discriminants, we want the last two terms $\sum_{\varrho}$ and $\sum_{\mathfrak{p}, m}$ in A. Weil's formula (2) to be both non-negative. Let us have a closer look at them.

1. The term $\sum_{\varrho} \Phi(\varrho)$ depends heavily on the zeros $\varrho$ of $\zeta_{k}$, and on $\Phi$ (defined in formula $(3)$ ).

Assuming the Generalized Riemann Hypothesis, the situation is fairly simple: $\sum_{\varrho} \Phi(\varrho)$ is non-negative if the Fourier transform $\widehat{F}$ of $F$ is nonnegative. Indeed, the zeros $\varrho=1 / 2+i t$ of $\zeta_{k}$ all have real part $1 / 2$ and $\Phi(\varrho)=\Phi(1 / 2+i t)=\widehat{F}(t)$ where $\widehat{F}(t):=\int_{-\infty}^{\infty} F(x) e^{i t x} d x$ is the Fourier transform of $F$.

Without the Generalized Riemann Hypothesis, assuming we do not have any further knowledge on the zeros, we want $\operatorname{Re} \Phi(s)$ to be non-negative on the whole region $0<\operatorname{Re} s<1$. Since $F$ is real and even, we have

$$
\operatorname{Re}(\Phi(\beta+i t))=\int_{-\infty}^{\infty} F(x) \cosh ((\beta-1 / 2) x) e^{i t x} d x
$$


and in particular for $\beta=0$ and $\beta=1$,

$$
\operatorname{Re}(\Phi(i t))=\operatorname{Re}(\Phi(1+i t))=\int_{-\infty}^{\infty} F(x) \cosh (x / 2) e^{i t x} d x
$$

By the Maximum-Modulus principle, if $\Phi$ has a moderate growth when $t$ grows to infinity inside the region $0<\operatorname{Re} s<1$, then the minimum of $\operatorname{Re} \Phi(s)$ will be achieved on the boundary, and if we write

$$
F(x)=\frac{f(x)}{\cosh (x / 2)},
$$

then $\sum_{\varrho} \Phi(\varrho)$ will be non-negative if the Fourier transform of $f$ is nonnegative.

2. The last term $\sum_{\mathfrak{p}, m}$ of A. Weil's formula (2) is easier to deal with, as all we have to do to make sure it is non-negative is to choose for $F$ and $f$ functions that are non-negative themselves.

Finally, things seem to work out quite well if we choose $F(x)=G(x / b)$ or $f(x)=g(x / b)$. The parameter $b$ then needs to be chosen for each degree $n$ to give the best possible bound. Currently, the best known bounds assuming the Generalized Riemann Hypothesis are obtained with A. M. Odlyzko's choice:

$$
G(x)= \begin{cases}(1-|x|) \cos (\pi x)+\frac{1}{\pi} \sin (\pi|x|) & \text { for }|x| \leq 1 \\ 0 & \text { for }|x|>1\end{cases}
$$

Without assuming the Generalized Riemann Hypothesis, the best choice is L. Tartar's:

$$
g(x)=9\left(\frac{\sin x-x \cos x}{x^{3}}\right)^{2}
$$

See A. M. Odlyzko [23] or G. Poitou [28], [29] for further information.

3.2. Upper bound for $\zeta_{k}(\sigma), \sigma>1$, for totally real number fields $k$. For starters, let us state precisely what we mean by A. Weil's formula for a totally real number field:

Proposition 3 (see [28, Propositions 4 and 5]). Let $F$ be a real-valued even function with $F(0)=1$, for which the following conditions hold:

(i) The integral $\int_{0}^{\infty} F(x) \cosh (x / 2) d x$ exists.

(ii) The function $F$ is of bounded variation, the value at each point being the average of the limit to the right and the limit to the left.

(iii) The function $(1-F(x)) / x$ is also of bounded variation.

(iv) Assuming the Generalized Riemann Hypothesis, the Fourier transform of $F$ is non-negative. Without this hypothesis, the Fourier transform of $f(x)=F(x) \cosh (x / 2)$ is non-negative. 
Then

$\log D_{k} \geq n \log \left(8 \pi e^{\gamma}\right)+n \pi / 2-n I_{n, n}(F)+2 \sum_{\mathfrak{p}, m} \frac{\log \mathrm{N}(\mathfrak{p})}{(\mathrm{N}(\mathfrak{p}))^{m / 2}} F(m \log \mathrm{N}(\mathfrak{p}))$ where $I_{n, n}(F)$ was defined in $(5)$.

Now suppose we take $F(x)=(1 / x) \exp (-(\sigma-1 / 2) x)$ for $x \geq \log 2$. Then

$$
\begin{aligned}
\sum_{\mathfrak{p}, m} \frac{\log \mathrm{N}(\mathfrak{p})}{(\mathrm{N}(\mathfrak{p}))^{m / 2}} F(m \log \mathrm{N}(\mathfrak{p})) & =\sum_{\mathfrak{p}, m} \frac{1}{m(\mathrm{~N}(\mathfrak{p}))^{m \sigma}} \\
& =-\sum_{\mathfrak{p}} \log \left(1-\frac{1}{(\mathrm{~N}(\mathfrak{p}))^{\sigma}}\right)=\log \zeta_{k}(\sigma) .
\end{aligned}
$$

If we could extend $F$ for $x<\log 2$ so that its Fourier transform is nonnegative and the other conditions of Proposition 3 hold, we would obtain

$$
\log D_{k}-n \log \left(8 \pi e^{\gamma}\right)-n \pi / 2+n I_{n, n}(F) \geq 2 \log \zeta_{k}(\sigma)
$$

and the upper bound

$$
\zeta_{k}(\sigma)<\left(\frac{D_{k}}{\exp \left(n C_{0}(F)\right)}\right)^{1 / 2}, \quad C_{0}(F)=\log (8 \pi)+\gamma+\pi / 2-I_{n, n}(F) .
$$

(Compare with (12).) However, we do not know how to determine whether the Fourier transform of a given function is non-negative. It is a delicate problem and no better results are known on the subject than those of R. P. Boas and M. Kac (see [3]). They gave necessary but no sufficient conditions for the Fourier transform to be non-negative.

1. We must proceed otherwise, using only functions $F$ and $f$ whose Fourier transforms are known to be non-negative beforehand. But if we do that, then we lose the natural relation (6) we had between the last term $\sum_{\mathfrak{p}, m}$ of A. Weil's formula (2) and $\log \zeta_{k}$. Thus we must introduce another step, and derive an inequality of the type

$$
\log \zeta_{k}(\sigma)<2 c_{1}(\sigma, F) \sum_{\mathfrak{p}, m} \frac{\log \mathrm{N}(\mathfrak{p})}{(\mathrm{N}(\mathfrak{p}))^{m / 2}} F(m \log \mathrm{N}(\mathfrak{p}))
$$

with $c_{1}(\sigma, F) \leq 1 / 2$ (important for our purpose, see Section 3.3). This will be possible if $F$ is chosen greater than the function $x \mapsto(1 / x) \exp (-(\sigma-1 / 2) x)$ for $x \geq \log 2$.

2. First, maybe the most restrictive, condition (i) in Proposition 3 states that $\int_{0}^{\infty} F(x) \cosh (x / 2) d x$ must exist. For the record, to get lower bounds for discriminants, A. M. Odlyzko chose functions with compact support, for which the integral trivially exists. Because of the first requirement, we cannot do that here. Upon careful analysis, we actually have very little choice for the behavior at infinity of $F$ given the first two requirements. 
3. Another condition is that the discriminant bound we get should be as good as possible, i.e. we want the choice of $F$ to make the constant $C_{0}(F)$ in (7) as large as possible. Numerical experimentation shows that this depends mostly on the form of $F$ near the origin. It also shows that it is hard to do better than A. M. Odlyzko in that respect (see [23]), the best form being $F(x)=G(x / b)$ with

$$
G(x)=(1-x) \cos (\pi x)+\frac{1}{\pi} \sin (\pi x) .
$$

4. Lastly, we want conditions (ii), (iii) and (iv) in Proposition 3 to hold.

After all that, it is indeed a wonder that we can find a function which behaves quite well for $1-4$. We have the following:

TheOREM 4. Let $k$ be a totally real number field of degree $n \geq 1$. Assume $b>0$ is given and set

$$
F_{b}(x)=\frac{1}{\left(1+(x / b)^{2}\right) \cosh (x / 2)}
$$

for which

$$
I_{n, n}\left(F_{b}\right)=\frac{2 \pi b}{n}+I(b) \quad \text { with } \quad I(b)=\int_{0}^{\infty} \frac{\left(1-F_{b}(x)\right) e^{x / 2}}{\sinh (x)} d x
$$

(see formula (5) for the definition of $I_{n, n}(F)$ ),

$$
c_{1}(\sigma, b)=\sup _{x \geq 2} \frac{-\log \left(1-x^{-\sigma}\right)}{2 \sum_{m \geq 1} \frac{\log x}{x^{m / 2}} F_{b}(m \log x)}<+\infty,
$$

and

$$
\begin{aligned}
C_{2}(b, n) & =\log \left(8 \pi e^{\gamma}\right)+\pi / 2-I_{n, n}\left(F_{b}\right) \\
& =\log \left(8 \pi e^{\gamma+\pi / 2}\right)-I(b)-2 \pi b / n .
\end{aligned}
$$

Then, for $\sigma>1$,

$$
\zeta_{k}(\sigma)<\left(\frac{D_{k}}{\exp \left(n C_{2}(b, n)\right)}\right)^{c_{1}(\sigma, b)} .
$$

Proof. Formula (5) gives

$$
I_{n, n}\left(F_{b}\right)=\frac{4}{n} \int_{0}^{\infty} F_{b}(x) \cosh (x / 2) d x+\int_{0}^{\infty} \frac{\left(1-F_{b}(x)\right) e^{x / 2}}{\sinh (x)} d x
$$

and since the first integral is equal to $\pi b / 2$, we obtain formula (9) easily.

We have

$$
\sum_{m \geq 1} \frac{\log x}{x^{m / 2}} F_{b}(m \log x) \geq \frac{\log x}{\sqrt{x}} F_{b}(\log x)=\frac{2 \log x}{(1+x)\left(1+(\log (x / b))^{2}\right)} .
$$


So for any $\sigma>1$,

$$
\frac{-\log \left(1-x^{-\sigma}\right)}{2 \sum_{m \geq 1} \frac{\log x}{x^{m / 2}} F_{b}(m \log x)}=O_{\sigma}\left(x^{1-\sigma} \log x\right) .
$$

We then have, by summing over $\mathrm{N}(\mathfrak{p})$,

$$
\begin{aligned}
\log \zeta_{k}(\sigma) & =-\sum_{\mathfrak{p}} \log \left(1-\frac{1}{(\mathrm{~N}(\mathfrak{p}))^{\sigma}}\right) \\
& <2 c_{1}(\sigma, b) \sum_{\mathfrak{p}, m} \frac{\log \mathrm{N}(\mathfrak{p})}{(\mathrm{N}(\mathfrak{p}))^{m / 2}} F_{b}(m \log \mathrm{N}(\mathfrak{p})) .
\end{aligned}
$$

We now check that the conditions of Proposition 3 hold for this choice of $F_{b}$. We have $F_{b}(0)=1, F_{b}$ is indeed even. The conditions (i)-(iii) trivially hold. The condition (iv) holds because the Fourier transform of $f(x)=$ $1 /\left(1+(x / b)^{2}\right)$ is $\widehat{f}=\int_{-\infty}^{\infty} f(t) e^{i t x} d t=\pi b e^{-b|t|}$, which is non-negative. We can then apply Proposition 3 to obtain

$$
\begin{aligned}
\log \zeta_{k}(\sigma) & <c_{1}(\sigma, b)\left(\log D_{k}-n \log \left(8 \pi e^{\gamma}\right)-n \pi / 2+n I_{n, n}\left(F_{b}\right)\right) \\
& =c_{1}(\sigma, b)\left(\log D_{k}-n C_{2}(b, n)\right) .
\end{aligned}
$$

As a closing remark, it is interesting to note that this rather trivial theorem is extremely powerful for this choice of $F$. The constant $C_{2}(b, n)$ is large and when $D_{k}$ happens to be close to its minimal values, the upper bound for the residue we will deduce will surpass any other known upper bound for residues. Also, numerically, the constant $c_{1}(\sigma, b)$ will be around 0.3 , which makes it even better.

3.3. Relationship between $\operatorname{Res} \zeta_{k}$ and $\zeta_{k}(\sigma)$. We will use:

Lemma 5 (see [22], [29] or [35, Lemma 11.11]). Suppose $\widetilde{\sigma}, \sigma>1$ satisfy $\tilde{\sigma} \geq 1+\sigma / \sqrt{7+4 \sqrt{2}}$ and $\tilde{\sigma} \geq\left(5+\sqrt{12 \sigma^{2}-5}\right) / 6$. Then, for any complex $\varrho$ in the vertical strip $0 \leq \operatorname{Re} \varrho<1$,

$$
\begin{aligned}
& \operatorname{Re}\left(\frac{1}{\sigma-\varrho}\right)+\operatorname{Re}\left(\frac{1}{\sigma-(1-\varrho)}\right) \\
& \quad \geq\left(\sigma-\frac{1}{2}\right)\left(\operatorname{Re}\left(-\frac{1}{(\widetilde{\sigma}-\varrho)^{2}}\right)+\operatorname{Re}\left(-\frac{1}{(\widetilde{\sigma}-(1-\varrho))^{2}}\right)\right) .
\end{aligned}
$$

Moreover, if $\widetilde{\sigma} \geq 1+(\sigma-1) / \sqrt{3}$, then (13) holds for any $\varrho$ of real part $1 / 2$.

Using this lemma, we prove

LemMa 6. Let $k$ be a totally real number field of degree $n \geq 1$. Set

$$
h(\sigma)=\pi^{-\sigma / 2} \Gamma(\sigma / 2) \quad(\sigma>1) .
$$

If $\zeta_{k}(s)$ has no real zero in the range $1 / 2<\beta<1$, set $E_{\sigma}=1$, if $\zeta_{k}(s)$ has at least one real zero in that range, set $E_{\sigma}=(1-\beta) /(\sigma-\beta)$ where $\beta$ is any 
of those zeros. In addition, set $\psi(\sigma)=\left(\Gamma^{\prime} / \Gamma\right)(\sigma)$, and for $\widetilde{\sigma}$ satisfying the conditions of Lemma 5, set

$$
c_{3}(\widetilde{\sigma}, n)=\frac{n}{4} \psi^{\prime}\left(\frac{\widetilde{\sigma}}{2}\right)-\frac{1}{\widetilde{\sigma}^{2}}-\frac{1}{(\widetilde{\sigma}-1)^{2}} .
$$

Then, for $\sigma>1$,

$$
\operatorname{Res} \zeta_{k}<E_{\sigma} \frac{\sigma(\sigma-1) \zeta_{k}(\sigma) D_{k}^{(\sigma-1) / 2} h^{n}(\sigma)}{\exp \left(\sigma(\sigma-1) c_{3}(\widetilde{\sigma}, n) / 2\right)} .
$$

Proof. The starting point is the following identity of H. M. Stark (which can be derived from the Weierstrass product of the entire function $\xi_{k}$ of order 1):

$$
\xi_{k}(s):=s(s-1)\left(\frac{D_{k}}{\pi^{n}}\right)^{s / 2} \Gamma(s / 2)^{n} \zeta_{k}(s)=e^{A} \prod_{\varrho}^{\prime}\left(1-\frac{s}{\varrho}\right)
$$

where $\varrho$ runs through the non-trivial zeros of $\zeta_{k}(s)$ and the prime indicates that $\varrho$ and $\varrho$ must be grouped together. Taking logarithmic derivatives, we obtain

$$
\frac{\xi_{k}^{\prime}(\sigma)}{\xi_{k}(\sigma)}=\sum^{\prime} \frac{1}{\sigma-\varrho}=\sum_{\operatorname{Im} \varrho \geq 0} \operatorname{Re}\left(\frac{1}{\sigma-\varrho}\right)
$$

where the sum on the right side is taken over all zeros $\varrho$ of $\zeta_{k}(s)$ with non-negative imaginary part. Following A. M. Odlyzko, we set

$$
Z(\sigma)=-\frac{\zeta_{k}^{\prime}(\sigma)}{\zeta_{k}(\sigma)} \quad \text { and } \quad Z_{1}(\sigma)=-\frac{d}{d \sigma} Z(\sigma)
$$

and notice that $Z(\sigma)>0$ and $Z_{1}(\sigma)>0$ for $\sigma>1$. Notice also that

$$
\begin{aligned}
\sum_{\operatorname{Im} \varrho \geq 0} \operatorname{Re}\left(-\frac{1}{(\sigma-\varrho)^{2}}\right) & =\left(\frac{\xi_{k}^{\prime}}{\xi_{k}}\right)^{\prime}(\sigma) \\
& =\frac{n}{4} \psi^{\prime}\left(\frac{\sigma}{2}\right)+Z_{1}(\sigma)-\frac{1}{\sigma^{2}}-\frac{1}{(\sigma-1)^{2}} .
\end{aligned}
$$

Let us now prove the lemma in the case where $\beta$ exists, for example. We have

$$
\begin{aligned}
& \left(\frac{\xi_{k}^{\prime}}{\xi_{k}}\right)^{\prime}(\sigma)-\frac{1}{\sigma-\beta} \geq\left(\frac{\xi_{k}^{\prime}}{\xi_{k}}\right)^{\prime}(\sigma)-\frac{1}{\sigma-\beta}-\frac{1}{\sigma-(1-\beta)} \\
& =\sum_{\substack{\varrho \neq \beta, 1-\beta \\
\operatorname{Im} \varrho \geq 0}} \operatorname{Re}\left(\frac{1}{\sigma-\varrho}\right)=\frac{1}{2} \sum_{\substack{\varrho \neq \beta, 1-\beta \\
\operatorname{Im} \varrho \geq 0}} \operatorname{Re}\left(\frac{1}{\sigma-\varrho}\right)+\operatorname{Re}\left(\frac{1}{\sigma-(1-\varrho)}\right) \\
& \quad\left(\text { since } \zeta_{k}(s)=\zeta_{k}(1-s)\right)
\end{aligned}
$$




$$
\geq \frac{1}{2}\left(\sigma-\frac{1}{2}\right) \sum_{\substack{\varrho \neq \beta, 1-\beta \\ \operatorname{Im} \varrho \geq 0}} \operatorname{Re}\left(-\frac{1}{(\widetilde{\sigma}-\varrho)^{2}}\right)+\operatorname{Re}\left(-\frac{1}{(\widetilde{\sigma}-(1-\varrho))^{2}}\right)
$$

(by Lemma 5)

$$
\geq\left(\sigma-\frac{1}{2}\right) \sum_{\operatorname{Im} \varrho \geq 0} \operatorname{Re}\left(-\frac{1}{(\widetilde{\sigma}-\varrho)^{2}}\right)
$$

(for $\varrho=\beta$ the right term of (13) is negative)

$$
\begin{aligned}
& =\left(\sigma-\frac{1}{2}\right)\left(\frac{n}{4} \psi^{\prime}\left(\frac{\tilde{\sigma}}{2}\right)+Z_{1}(\widetilde{\sigma})-\frac{1}{\widetilde{\sigma}^{2}}-\frac{1}{(\widetilde{\sigma}-1)^{2}}\right) \quad(\text { by }(17)) \\
& \geq\left(\sigma-\frac{1}{2}\right)\left(\frac{n}{4} \psi^{\prime}\left(\frac{\tilde{\sigma}}{2}\right)-\frac{1}{\widetilde{\sigma}^{2}}-\frac{1}{(\widetilde{\sigma}-1)^{2}}\right)=\left(\sigma-\frac{1}{2}\right) c_{3}(\widetilde{\sigma}, n) .
\end{aligned}
$$

If we now sum this inequality

$$
\frac{\xi_{k}^{\prime}(\sigma)}{\xi_{k}(\sigma)}-\frac{1}{\sigma-\beta} \geq\left(\sigma-\frac{1}{2}\right) c_{3}(\widetilde{\sigma}, n)
$$

from 1 to $\sigma$, we obtain

$$
\log \frac{\xi_{k}(\sigma)}{\xi_{k}(1)}+\log E_{\sigma} \geq\left(\frac{\sigma^{2}-\sigma}{2}\right) c_{3}(\widetilde{\sigma}, n)
$$

which concludes the proof.

3.4. Upper bounds for $\operatorname{Res} \zeta_{k}$. In Lemma 6, we have an upper bound for $\operatorname{Res} \zeta_{k}$ in which $\zeta_{k}(\sigma)$ occurs. Combined with the upper bound for $\zeta_{k}(\sigma)$ we got in Theorem 4, we have an effective upper bound for the residue $\operatorname{Res} \zeta_{k}$. It is convenient to write this bound in the following form:

TheOREM 7 (cf. [11, Theorem $\left.1^{\prime}\right]$ ). Let $k$ be a totally real number field of degree $n \geq 1$, let $\sigma>1$ and $b>0$ be given, let $c_{1}=c_{1}(\sigma, b)$ and $C_{2}=C_{2}(b, n)$ be as in Theorem 4, let $h(\sigma)=\pi^{-\sigma / 2} \Gamma(\sigma / 2)$ and $c_{3}=c_{3}(\widetilde{\sigma}, n)$ be as in Lemma 6, let

$$
c_{4}=c_{4}(\sigma, b)=c_{1}(\sigma, b)+(\sigma-1) / 2,
$$

and let $C_{5}=C_{5}(n, b, \sigma, \tilde{\sigma})$ be defined by

$$
C_{5}=\exp \left(\frac{1}{c_{4}}\left(c_{1} C_{2}+\frac{\sigma(\sigma-1)}{2} \cdot \frac{c_{3}}{n}-\frac{1}{n} \log (\sigma(\sigma-1))-\log h(\sigma)\right)\right) .
$$

Finally, let $E_{\sigma}$ be as in Lemma 6. Then

$$
\operatorname{Res} \zeta_{k} \leq E_{\sigma}\left(\frac{D_{k}}{C_{5}(n, b, \sigma, \tilde{\sigma})^{n}}\right)^{c_{4}(\sigma, b)} .
$$

Possible values for $C_{5}(n, b, \sigma, \widetilde{\sigma})$ and $c_{4}(\sigma, b)$ for small degrees are given in Table 4 below. 
Before we proceed any further, let us examine the behavior of $n \mapsto$ $C_{5}(n, b, \sigma, \widetilde{\sigma})$ for given $b, \sigma$ and $\widetilde{\sigma}$. We first notice that only $C_{2}=C_{2}(b, n)$ and $c_{3}=c_{3}(\widetilde{\sigma}, n)$ depend on $n$. As can be seen in Lemma $6, n \mapsto c_{3}(\widetilde{\sigma}, n) / n$ is increasing for given $\widetilde{\sigma}$. Moreover, we have

$$
C_{2}(b, n)=\log \left(8 \pi e^{\gamma+\pi / 2}\right)-2 \pi b / n-I(b) .
$$

Thus, if we choose $b>0$ and $\sigma>1$ such that

$$
2 \pi b c_{1}(\sigma, b)+\log (\sigma(\sigma-1)) \geq 0,
$$

then the function $n \mapsto C_{5}(n, b, \sigma, \widetilde{\sigma})$ will be increasing for given $b, \sigma$ and $\widetilde{\sigma}$.

We now fix $m$, and we want to find $b, \sigma$ (for which (21) holds) and $\widetilde{\sigma}$ (for which the conditions of Lemma 5 hold) so that $C_{5}(m, b, \sigma, \widetilde{\sigma})$ is as large as possible and $c_{4}(\sigma, b)$ is as small as possible. Notice that for such a choice of $b, \sigma$ and $\widetilde{\sigma}$, we have $C_{5}(n, b, \sigma, \widetilde{\sigma}) \geq C_{5}(m):=C_{5}(m, b, \sigma, \widetilde{\sigma})$ and

$$
\operatorname{Res} \zeta_{k} \leq E_{\sigma}\left(\frac{D_{k}}{C_{5}(m)^{n}}\right)^{c_{4}(m)}
$$

for any totally real number field $k$ of degree $n \geq m$.

To compute these values, for a given $m$, we first optimize $b$ to have the largest possible $C_{2}(b, m)$ ( $b$ determines $I(b)$ as in Theorem 4$)$. Then we take $\sigma$ as close to 1 as possible while keeping $c_{1}(\sigma, b)$ fairly low. Finally, we optimize $\widetilde{\sigma}$ so that $c_{3}(\widetilde{\sigma}, m)$ is maximal. For further details, we refer to $[2]$.

With or without assuming the Generalized Riemann Hypothesis, the only difference is in the optimization of $\widetilde{\sigma}$. Rather interestingly, the unconditional restrictions only come into play for degrees $n \geq 86$. We will only use degrees $n=87$ and $n=88$ while assuming the Generalized Riemann Hypothesis, so it is understood that those two lines in the table are under this assumption. However, we will only consider the degrees $n \geq 90$ unconditionally, and so the corresponding lines are under no particular hypothesis. The bounds in the following table could be slightly improved assuming the Generalized Riemann Hypothesis for $n \geq 90$ :

Table 4. Possible constants for upper bounds of residues

\begin{tabular}{cccccc}
\hline$m$ & $b$ & $\sigma$ & $\tilde{\sigma}$ & $c_{4}(\sigma, b)$ & $C_{5}(m, b, \sigma, \tilde{\sigma})$ \\
\hline 134 & 5.89464 & 1.00755 & 1.28322 & 0.35501 & 43.2532 \\
133 & 5.87605 & 1.00760 & 1.28324 & 0.35517 & 43.1904 \\
130 & 5.81968 & 1.00774 & 1.28327 & 0.35565 & 42.9983 \\
120 & 5.62499 & 1.00825 & 1.28342 & 0.35739 & 42.3126 \\
110 & 5.41864 & 1.00884 & 1.28359 & 0.35942 & 41.5469 \\
100 & 5.19876 & 1.00955 & 1.28379 & 0.36181 & 40.6841 \\
90 & 4.96299 & 1.01042 & 1.28403 & 0.36468 & 39.7013 \\
\hline
\end{tabular}


Table 4 (cont.)

\begin{tabular}{cccccc}
\hline$m$ & $b$ & $\sigma$ & $\tilde{\sigma}$ & $c_{4}(\sigma, b)$ & $C_{5}(m, b, \sigma, \tilde{\sigma})$ \\
\hline 88 & 4.91367 & 1.01061 & 1.28113 & 0.36532 & 39.4878 \\
87 & 4.88871 & 1.01071 & 1.28250 & 0.36565 & 39.3786 \\
\hline 83 & 4.78685 & 1.01114 & 1.28823 & 0.36705 & 38.9255 \\
82 & 4.76086 & 1.01125 & 1.28972 & 0.36742 & 38.8079 \\
80 & 4.70820 & 1.01148 & 1.29280 & 0.36819 & 38.5671 \\
70 & 4.43018 & 1.01284 & 1.31019 & 0.37261 & 37.2380 \\
60 & 4.12301 & 1.01463 & 1.33188 & 0.37837 & 35.6488 \\
50 & 3.77801 & 1.01713 & 1.36007 & 0.38624 & 33.6977 \\
40 & 3.38148 & 1.02087 & 1.39886 & 0.39776 & 31.2141 \\
30 & 2.90979 & 1.02713 & 1.45730 & 0.41652 & 27.8811 \\
27 & 2.74769 & 1.02993 & 1.48153 & 0.42476 & 26.6307 \\
25 & 2.63277 & 1.03219 & 1.50033 & 0.43133 & 25.7097 \\
24 & 2.57300 & 1.03346 & 1.51070 & 0.43503 & 25.2189 \\
\hline
\end{tabular}

Any bound from this table still holds for a totally real number field of degree $n \geq m$, but of course a better bound can be obtained in that case. As we have outlined, we choose $b, \sigma$ and $\widetilde{\sigma}$ depending on $m$ only, and thus it is possible to reformulate the previous theorem:

THEOREM 8. Let $k$ be a totally real number field of degree $n \geq m$ and let $E_{\sigma}$ be as in Lemma 6 . Then

$$
\operatorname{Res} \zeta_{k} \leq E_{\sigma}\left(\frac{D_{k}}{C_{5}(m)^{n}}\right)^{c_{4}(m)}
$$

where $c_{4}(m)$ and $C_{5}(m)$ are given in the following table:

Table 5. Upper bounds for residues depending on $m$ only

\begin{tabular}{cccccccc}
\hline$m$ & 24 & 25 & 27 & 30 & 40 & 50 & 60 \\
$c_{4}(m)$ & 0.43503 & 0.43133 & 0.42476 & 0.41652 & 0.39776 & 0.38624 & 0.37837 \\
$C_{5}(m)$ & 25.2189 & 25.7097 & 26.6307 & 27.8811 & 31.2141 & 33.6977 & 35.6488 \\
\hline$m$ & 70 & 80 & 82 & 83 & 87 & 88 & \\
$c_{4}(m)$ & 0.37261 & 0.36819 & 0.36742 & 0.36705 & 0.36565 & 0.36532 & \\
$C_{5}(m)$ & 37.2380 & 38.5671 & 38.8079 & 38.9255 & 39.3786 & 39.4878 & \\
\hline$m$ & 90 & 100 & 110 & 120 & 130 & 133 & 134 \\
$c_{4}(m)$ & 0.36468 & 0.36181 & 0.35942 & 0.35739 & 0.35565 & 0.35517 & 0.35501 \\
$C_{5}(m)$ & 39.7013 & 40.6841 & 41.5469 & 42.3126 & 42.9983 & 43.1904 & 43.2532 \\
\hline
\end{tabular}

3.5. Upper bound for $\operatorname{Res} \zeta_{k}$ for high discriminants. When the discriminant is much higher than Odlyzko's bounds, the upper bound for $\operatorname{Res} \zeta_{k}$ we have obtained from A. Weil's formula is not good enough. We must then use another bound, for example: 
TheOREM 9. Let $k$ be a number field of degree $n>1$.

1. (See [17, Theorem 1].) We have

$$
\operatorname{Res} \zeta_{k} \leq e\left(\frac{e \log D_{k}}{2 n}\right)^{n-1} \text {. }
$$

2. (See [18, Theorem 1].) Moreover, $1 / 2<\beta<1$ and $\zeta_{k}(\beta)=0$ imply

$$
\operatorname{Res} \zeta_{k} \leq(1-\beta)\left(\frac{e \log D_{k}}{2 n}\right)^{n} .
$$

Regarding our objective, this upper bound for the residue is used for $n \leq 23$ under the Generalized Riemann Hypothesis in the normal case, for $n \leq 24$ in the non-normal case, and for $n \leq 26$ without assuming the Generalized Riemann Hypothesis.

\section{Explicit lower bounds for residues of zeta functions of normal} number fields assuming the Generalized Riemann Hypothesis. The aim of this section is to prove the following explicit conditional lower bound for residues:

Theorem 10. Assume the Generalized Riemann Hypothesis. Then, for any normal number field $K$, we have the lower bound

$$
\operatorname{Res} \zeta_{K} \geq \frac{1}{\left(2 e^{\gamma}+o(1)\right) \log \log D_{K}}
$$

where $o(1)$ is an effective error term decreasing towards zero as $D_{K}$ increases towards infinity.

This is a particular case of a result of J. Buchmann and H. C. Williams (see [4]). They derive a similar one $\left(\log \log D_{k}\right) \operatorname{Res} \zeta_{k} \geq C$, in the nonnormal case, but the effective $C$ that would result is too large for our purpose.

We have

$$
\operatorname{Res} \zeta_{K}=\lim _{s \rightarrow 1}(s-1) \zeta_{K}(s)=\lim _{s \rightarrow 1}\left(\zeta_{K} / \zeta\right)(s)=\prod_{p \in P} E(p)
$$

where $P$ is the set of all prime numbers $p \geq 2$ and

$$
E(p)=\left(1-p^{-1}\right) \prod_{\mathfrak{p} \mid p}\left(1-\mathrm{N}(\mathfrak{p})^{-1}\right)^{-1}
$$

is the eulerian factor associated with $p$ and $\zeta_{K} / \zeta$. We write

$$
\operatorname{Res} \zeta_{K}=M(Q) R(Q) T(Q)
$$


with

$$
M(Q)=\prod_{p \leq Q} E(p), \quad R(Q)=\prod_{\substack{p>Q \\ p \text { ramified }}} E(p), \quad T(Q)=\prod_{\substack{p>Q \\ p \text { non-ramified }}} E(p) .
$$

The whole difficulty is to give a subtle estimate for the tail $T(Q)$ so that we can choose a reasonably high value for $Q$. Once this is done properly, we will simply use trivial lower bounds for the main term $M(Q)$ and the ramified term $R(Q)$.

4.1. Bounds for the tail $T(Q)$

LEMMA 11. Let $K$ be a normal number field of degree $n$. Set

$$
f_{K}(t)=\left(\frac{1}{\pi \log t}+\frac{5.3}{\log ^{2} t}\right) \log D_{K}+(n+1)\left(\frac{1}{2 \pi}+\frac{2}{\log t}\right) .
$$

Then, assuming the Generalized Riemann Hypothesis, we have

$$
|\log T(Q)| \leq \frac{4+3 \log Q}{\sqrt{Q}} f_{K}(Q)+\frac{n}{Q} .
$$

In particular, for $a>2$ we have $\lim _{D_{K} \rightarrow \infty} \log T\left(\left(\log D_{K}\right)^{a}\right)=0$.

Proof. Each eulerian factor $E(p)$ depends on the norm $\mathrm{N}(\mathfrak{p})=p^{f}$ of the ideals above $p$ and if we set

$$
a_{1}(p)= \begin{cases}1 & \text { if } f>1 \\ 1-n & \text { if } f=1\end{cases}
$$

then

$$
\frac{E(p)}{(1-1 / p)^{a_{1}(p)}}= \begin{cases}1 & \text { if } f=1 \\ \left(1-1 / p^{f}\right)^{-n / f} & \text { if } f>1\end{cases}
$$

Hence,

$$
\begin{aligned}
\log T(Q) & =\sum_{\substack{p>Q \\
p \text { non-ram }}} a_{1}(p) \log \left(1-\frac{1}{p}\right)-\sum_{\substack{p>Q \\
p \text { non-ram } \\
f_{p}>1}} \frac{n}{f_{p}} \log \left(1-\frac{1}{p^{f_{p}}}\right) \\
& =-\sum_{\substack{p>Q \\
p \text { non-ram }}} \frac{a_{1}(p)}{p}+\varrho(Q)
\end{aligned}
$$

where

(23) $\varrho(Q)=\sum_{\substack{p>Q \\ p \text { non-ram }}} a_{1}(p)\left(\frac{1}{p}+\log \left(1-\frac{1}{p}\right)\right)-\sum_{\substack{p>Q \\ p \text { non-ram } \\ f_{p}>1}} \frac{n}{f_{p}} \log \left(1-\frac{1}{p^{f_{p}}}\right)$.

LEMMA 12. $n \geq 2$ and $Q \geq n$ imply $|\varrho(Q)| \leq n / Q$. 
Proof. We have

$$
\begin{aligned}
|\varrho(Q)| & \leq(n-1) \sum_{p>Q}\left(-\frac{1}{p}-\log \left(1-\frac{1}{p}\right)\right)+\frac{n}{2} \sum_{p>Q}-\log \left(1-\frac{1}{p^{2}}\right) \\
& \leq(n-1) \sum_{p>Q} \frac{1}{2 p^{2}} \cdot \frac{1}{1-1 / p}+\frac{n}{2} \sum_{p>Q} \frac{1}{p^{2}} \cdot \frac{1}{1-1 / p^{2}} \\
& \leq \frac{n-1}{1-1 /(n+1)} \sum_{p>Q} \frac{1}{2 p^{2}}+\frac{n}{1-1 /(n+1)^{2}} \sum_{p>Q} \frac{1}{2 p^{2}} \quad(\text { for } p>Q \geq n) \\
& \leq\left(\frac{n^{2}-1}{n}+\frac{n^{3}+2 n^{2}+n}{n(n+2)}\right) \frac{1}{2 Q} \\
& =\left(2 n-\frac{2}{n(n+2)}\right) \frac{1}{2 Q} \leq \frac{n}{Q} .
\end{aligned}
$$

It remains to estimate the sum $\sum_{p>Q} a_{1}(p) / p$. We shall use the method developed by G. Cornell and L. C. Washington [6] for cyclotomic fields. We wish to perform an Abel transform on the sum, so we set

$$
A(t)=\sum_{p<t} a_{1}(p), \quad \pi_{1}(t)=\sum_{\substack{p<t \\ f=1}} 1,
$$

and we write

$$
A(t)=\pi(t)-n \pi_{1}(t)=\pi(t)-\operatorname{li}(t)+\operatorname{li}(t)-n \pi_{1}(t) .
$$

At this point, we use J. Oesterlé's explicit form of J. C. Lagarias and A. M. Odlyzko's results, according to which, assuming the Generalized Riemann Hypothesis, we have (see [24, Théorème 3])

$$
\left|\pi_{1}(t)-\frac{1}{n} \operatorname{li}(t)\right| \leq \frac{1}{n} C_{K}(t) \sqrt{t} \log t
$$

where

$$
C_{K}(t)=\left(\frac{1}{\pi \log t}+\frac{5.3}{\log ^{2} t}\right) \log D_{K}+n\left(\frac{1}{2 \pi}+\frac{2}{\log t}\right)
$$

which in the particular case $K=\mathbb{Q}$ gives

$$
|\pi(t)-\operatorname{li}(t)| \leq\left(\frac{1}{2 \pi}+\frac{2}{\log t}\right) \sqrt{t} \log t
$$

Hence

$$
|A(t)| \leq f_{K}(t) \sqrt{t} \log t .
$$

Since $t \mapsto f_{K}(t)$ is a decreasing function of $t>1$, we obtain

$$
\left|\sum_{p>Q} \frac{a_{1}(p)}{p}\right|=\left|\int_{Q}^{\infty} \frac{1}{t} d A(t)\right|=\left|\frac{A(Q)}{Q}+\int_{Q}^{\infty} \frac{A(t)}{t^{2}} d t\right|
$$




$$
\begin{aligned}
& \leq \frac{|A(Q)|}{Q}+f_{K}(Q) \int_{Q}^{\infty} \frac{\log t}{t^{3 / 2}} d t \\
& \leq f_{K}(Q) \frac{\log Q}{\sqrt{Q}}+f_{K}(Q) \frac{4+2 \log Q}{\sqrt{Q}}
\end{aligned}
$$

or

$$
\left|\sum_{p>Q} \frac{a_{1}(p)}{p}\right| \leq f_{K}(Q) \frac{4+3 \log Q}{\sqrt{Q}} .
$$

Combining (22)-(24), we get the desired result. The last assertion of the lemma comes from the fact that $n=O\left(\log D_{K}\right)$.

4.2. Proof of Theorem 10. Now that the tail $T(Q)$ of the eulerian product is under control, it remains to obtain lower bounds for the main term $M(Q)$ and the ramified term $R(Q)$. For $Q \geq 285$, we have

$$
M(Q)=\prod_{p \leq Q} E(p) \geq \prod_{p \leq Q}\left(1-\frac{1}{p}\right) \geq \frac{1}{e^{\gamma} \log Q}\left(1-\frac{1}{2 \log ^{2} Q}\right)
$$

(by $[30,(3.25)])$. For the ramified term $R(Q)$, since the ramified primes greater than $Q$ divide $D_{K}$, there are at most $\log D_{K} / \log Q$ such primes and

$$
R(Q)=\prod_{\substack{p>Q \\ p \text { ramified }}} E(p) \geq \prod_{\substack{p>Q \\ p \text { ramified }}}\left(1-\frac{1}{p}\right) \geq(1-1 / Q)^{\log D_{K} / \log Q}
$$

Combining (25), (26) and Lemma 11, with $Q=\left(\log D_{K}\right)^{a} \geq 285$, we conclude that for $a>2$ we have

$$
\liminf _{D_{K} \rightarrow \infty}\left(\log \log D_{K}\right) \operatorname{Res} \zeta_{K} \geq \frac{1}{a e^{\gamma}},
$$

which proves Theorem 10.

However, this theorem only provides an asymptotic lower bound for $\operatorname{Res} \zeta_{K}$ and we will need the following explicit result:

COROLlary 13. Let $m \geq 1$ and $\varrho>e$ be given. There exists an effective $c_{6}(m, \varrho)$ such that, for any normal number field $K$ of degree $n \geq m$ and of root discriminant $\varrho_{K}=D_{K}^{1 / n} \geq \varrho$,

$$
\operatorname{Res} \zeta_{K} \geq \frac{1}{c_{6}(m, \varrho) \log \log D_{K}} .
$$

The following table gives the $a$ and $c_{6}(m, \varrho)$ that we use to establish Theorem 2: 
Table 6. Conditional lower bounds for the residues

\begin{tabular}{cccccccc}
\hline$m$ & 166 & 164 & 140 & 120 & 100 & 80 & 60 \\
$\varrho$ & 54.88 & 54.76 & 65.76 & 81.93 & 114.5 & 204.0 & 729.3 \\
$a$ & 3.27897 & 3.28114 & 3.28825 & 3.29382 & 3.29534 & 3.28809 & 3.25254 \\
$c_{6}(m, \varrho)$ & 6.43518 & 6.44025 & 6.46426 & 6.48259 & 6.49536 & 6.49202 & 6.43824 \\
\hline$m$ & 48 & 46 & 40 & 30 & 20 & 10 & \\
$\varrho$ & 5252 & 6354 & 6499 & 6875 & 7653 & 10250 & \\
$a$ & 3.20022 & 3.19990 & 3.22302 & 3.27400 & 3.35472 & 3.52317 & \\
$c_{6}(m, \varrho)$ & 6.33092 & 6.33214 & 6.38811 & 6.51149 & 6.70664 & 7.11282 & \\
\hline
\end{tabular}

Proof. Set

$$
g_{K}(Q)=\frac{\exp \left(\frac{4+3 \log Q}{\sqrt{Q}} f_{K}(Q)+\frac{n}{Q}\right)}{\left(1-\frac{1}{2 \log ^{2} Q}\right)\left(1-\frac{1}{Q}\right)^{\log D_{K} / \log Q}} .
$$

Combining (25), (26) and Lemma 11, we get

$$
\operatorname{Res} \zeta_{K} \geq \frac{1}{a e^{\gamma} g\left(a, \varrho_{K}, n\right) \log \log D_{K}}
$$

where $g\left(a, \varrho_{K}, n\right)=g_{K}\left(\left(n \log \varrho_{K}\right)^{a}\right)$ is a function of $a, \varrho_{K}$ and $n$ only. Since the functions $\varrho_{K} \mapsto g\left(a, \varrho_{K}, n\right)$ and $n \mapsto g(a, \varrho, n)$ are decreasing, we have

$$
a e^{\gamma} g\left(a, \varrho_{K}, n\right) \leq a e^{\gamma} g(a, \varrho, n) \leq a e^{\gamma} g(a, \varrho, m) .
$$

The function $a \mapsto g(a, \varrho, m)$ is decreasing as well. As a result, for given $m$ and $\varrho$, we can find the optimal value of $a$ which minimizes the product $a e^{\gamma} g(a, \varrho, m)$. This proves the existence of an absolute constant $c_{6}(m, \varrho)$ for which we have for any normal number field of degree $n \geq m$ the lower bound

$$
\operatorname{Res} \zeta_{K} \geq \frac{1}{c_{6}(m, \varrho) \log \log D_{K}} .
$$

REMARK. To obtain for given $m$ the explicit values of the constant $c_{6}(m, \varrho)$ in Corollary 13, we could have chosen for $\varrho$ Odlyzko's lower bound $\varrho_{m}$ for root discriminants of CM-fields of degree $m$, i.e. Odlyzko's lower bound $\varrho_{m}$ for root discriminants of totally real fields of degree $m$. This would have yielded, for each $m$ appearing in Table 6 , a constant $c_{6}(m, \varrho)=$ $c_{6}\left(m, \varrho_{m}\right)=c_{6}(m)$ depending on $m$ only, but slightly larger than the one we give in Table 6 . This would have given for the first case of Theorem 2 bounds not as good as the ones we give there. To get the best possible bounds for the first case of Theorem 2 , we need, for given $m$, to compute $c_{6}(m, \varrho)$ with $\varrho$ precisely equal to the lower bound for root discriminants of CM-fields with relative class number one of degree $m$, lower bound given in the first case of Theorem 2. Clearly, there is a small problem of optimization to choose those values of $\varrho$, but there is no problem to see that the values we give in Table 6 will prove Theorem 2 . 
4.3. Non-normal case. In the non-normal case, we can obtain a similar bound to the one we obtained in Section 4.2, but we must then work inside the normal closure of $L$ of $K$, and we get bounds of the form $\operatorname{Res} \zeta_{K}>$ $1 /\left(\left(2 e^{\gamma}+o(1)\right) \log \log D_{L}\right)$. Unfortunately, in the general case, the only upper bound we have for $D_{L}$ is $D_{L} \leq D_{K}^{(2 n-1) !}$, which makes those lower bounds for $\operatorname{Res} \zeta_{K}$ rather worthless for our purpose. We need something better. The following lower bound for residues has been communicated to us by J. Oesterlé (see the proof in Appendix):

TheOREm 14 (J. Oesterlé). For any number field $K$ different from $\mathbb{Q}$ for which the Riemann Hypothesis for $\zeta_{K}(s)$ holds true we have

$$
\operatorname{Res} \zeta_{K} \geq \frac{e^{-3 / 2}}{\sqrt{\log D_{K}}} \exp \left(\frac{-1}{\sqrt{\log D_{K}}}\right) .
$$

5. Unconditional explicit lower bounds for residues of zeta functions of CM-fields. If we do not assume the Generalized Riemann Hypothesis, the method is completely different, as we have no analog of the tools used in the preceding section. The important point is to control the zeros of $\zeta_{K}$. For the following, $K$ is simply a totally imaginary field of discriminant $D_{K}$ and of degree $2 n$.

Lemma 15 (see [14, Lemma 15]). Set $\kappa=(2+\sqrt{3}) / 4=0.933 \ldots$ Then $\zeta_{K}(s)$ has at most two zeros counted with multiplicity in the range 1 $1 /\left(\kappa \log D_{K}\right) \leq s<1$.

Once we have this, we use a theorem of [19], which can be slightly rewritten as:

TheOrem 16. Let $m \geq 1$ be a positive integer. Let $c>0$ be given. There exists an effective $\varrho(2 m, c)$ such that for any totally imaginary number field $K$ of degree $2 n \geq 2 m$ and root discriminant $\varrho_{K}:=D_{K}^{1 /(2 n)} \geq \varrho(2 m, c)$ we have

$$
\operatorname{Res} \zeta_{K} \geq \frac{1}{c e^{1 /(2 c)} \log D_{K}}
$$

if $\zeta_{K}\left(1-1 /\left(c \log D_{K}\right)\right) \leq 0$, and

$$
\operatorname{Res} \zeta_{K} \geq \frac{1-\beta}{2 e^{1 /(2 c)}}
$$

if $\zeta_{K}(\beta) \leq 0$ and $1-1 /\left(c \log D_{K}\right) \leq \beta<1$.

Moreover, for $c=\kappa=(2+\sqrt{3}) / 4$ and $m \geq 5$, we may take $\varrho(2 m, c)$ $=2 \pi^{2}$.

We remark that in the most favorable case, $\zeta_{K}\left(1-\left(c \log D_{K}\right)^{-1}\right) \leq 0$ and we get 


$$
\operatorname{Res} \zeta_{K} \geq \frac{1}{c e^{1 /(2 c)} \log D_{K}} .
$$

We will see in Section 7 that this "most favorable" case is in fact the worse thing that can happen. So we can already give the lower bound we will use:

$$
\operatorname{Res} \zeta_{K} \geq \frac{1}{\kappa e^{1 /(2 \kappa)} \log D_{K}} .
$$

\section{A bound for Siegel's zeros of zeta functions of imaginary} quadratic fields. The aim of this section is to prove the following effective upper bound on Siegel's zeros of zeta functions of imaginary quadratic fields (cf. [33, Lemma 8]):

THEOREM 17. Let $c>0$ be given and let $F$ range over the imaginary quadratic number fields. There exists an effective constant $D_{c}$ such that if $D_{F}>D_{c}$ then $\zeta_{F}(s)<0$ in the range $1-c / \sqrt{D_{F}} \leq s \leq 1$. In particular, for all imaginary quadratic fields $F$, we have $\zeta_{F}(s)<0$ in the range $1-$ $6 /\left(\pi \sqrt{D_{F}}\right) \leq s<1$.

It is possible to derive explicit bounds from the theoretical bounds of Pintz [27], or of Goldfeld [8], or of Goldfeld and Schinzel [9]. However, our bounds are better and easier to obtain. Notice that J. Hoffstein used our last assertion on page 46 of [11], but the paper of his he was referring to never appeared!

From now on, we let $F=\mathbb{Q}(\sqrt{-d})$ range over the imaginary quadratic fields of discriminants $-d<-4$. We let $\chi_{-d}$ denote the primitive quadratic Dirichlet character modulo $d$ associated with $F$ and recall that we have the factorization $\zeta_{F}(s)=\zeta(s) L\left(s, \chi_{-d}\right)$. We then let $h(-d)$ denote the class number of $F$ and $Q(x, y)=a x^{2}+b x y+c y^{2}$ will always stand for a primitive reduced binary quadratic form of discriminant $-d=b^{2}-4 a c$. Recall that there are $h(-d)$ reduced binary quadratic forms of discriminant $-d$ and that if $Q$ is reduced then $1 \leq a \leq \sqrt{d / 3}$ and $-a<b \leq a$. The symbol $\sum_{a, b, c}$ will stand for sums over the $h(-d)$ reduced binary quadratic forms $Q(x, y)=a x^{2}+b x y+c y^{2}$ of discriminant $-d<-4$ and for such a form we set

$$
\zeta_{Q}(s)=\sum_{(m, n) \neq(0,0)}\left(\frac{a}{\sqrt{d}} n^{2}+\frac{b}{\sqrt{d}} m n+\frac{c}{\sqrt{d}} m^{2}\right)^{-s} .
$$

\subsection{Explicit bounds for the second derivatives of Dirichlet $L$-functions}

Lemma 18. Let $c>0$ be given and let $\chi$ range over the primitive Dirichlet characters of conductors $f_{\chi} \geq 4 c^{2}$ (which implies $1 / 2 \leq 1-c / \sqrt{f_{\chi}} \leq 1$ ). Then

$$
\left|L^{\prime \prime}(s, \chi)\right| \leq \frac{1}{24}(1+o(1)) \log ^{3} f_{\chi}
$$


in the range $1-c / \sqrt{f_{\chi}} \leq s \leq 1$ where $o(1)$ is an explicit error term which does not depend on $s$ and approaches 0 as $f_{\chi}$ goes to infinity. In particular, in the range $1-6 /\left(\pi \sqrt{f_{\chi}}\right) \leq s \leq 1$ we have

$$
\left|L^{\prime \prime}(s, \chi)\right| \leq \begin{cases}\frac{1}{3} \log ^{3} f_{\chi} & \text { if } f_{\chi} \geq 1108 \\ 0.3 \log ^{3} f_{\chi} & \text { if } f_{\chi} \geq 1775 \\ \frac{1}{4} \log ^{3} f_{\chi} & \text { if } f_{\chi} \geq 4692 \\ \frac{1}{5} \log ^{3} f_{\chi} & \text { if } f_{\chi} \geq 23393\end{cases}
$$

The reason why we state this upper bound with such precision is because in the end it will be the main error term in the lower bound for $1-\beta$. So getting the best possible bound will have a significant impact on the end result.

Proof. Fix an integer $B \geq e^{2}$. Then $t \mapsto\left(\log ^{2} t\right) / t$ is decreasing in the range $t \geq B$. Set $A_{\chi}=\sqrt{f_{\chi}} \log f_{\chi}$ and assume $f_{\chi} \geq 128$, which implies $A_{\chi} \geq e^{4}$. This ensures that $t \mapsto\left(\log ^{2} t\right) / t^{s}$ is decreasing in the range $t \geq A_{\chi}$, for any $s$ in the range $1 / 2 \leq s \leq 1$. Assume also that $f_{\chi}$ is large enough to guarantee $A_{\chi} \geq B$ and set $A=\left[A_{\chi}\right]=$ the greatest integer less than or equal to $A_{\chi}$. Hence $B \leq A$. Set $X(n)=\sum_{k=A+1}^{n} \chi(k)$, and recall that $|X(n)| \leq A_{\chi}$ (Pólya-Vinogradov's bound). For $1-c / \sqrt{f_{\chi}} \leq s \leq 1$, we have

$$
\begin{aligned}
L^{\prime \prime}(s, \chi)= & \sum_{n=1}^{B} \frac{\chi(n) \log ^{2} n}{n^{s}}+\sum_{n=B+1}^{A} \frac{\chi(n) \log ^{2} n}{n^{s}} \\
& +\sum_{n \geq A+1} X(n)\left(\frac{\log ^{2} n}{n^{s}}-\frac{\log ^{2}(n+1)}{(n+1)^{s}}\right)
\end{aligned}
$$

and

$$
\frac{1}{n^{s}}=\frac{n^{1-s}}{n} \leq \frac{A_{\chi}^{c / \sqrt{f_{\chi}}}}{n} \quad \text { for } n \leq A
$$

Hence we obtain

$$
\begin{aligned}
\left|L^{\prime \prime}(s, \chi)\right| \leq & A_{\chi}^{c / \sqrt{f_{\chi}}}\left(\sum_{n=1}^{B} \frac{\log ^{2} n}{n}+\sum_{n=B+1}^{A} \frac{\log ^{2} n}{n}\right) \\
& +A_{\chi} \sum_{n \geq A+1}\left(\frac{\log ^{2} n}{n^{s}}-\frac{\log ^{2}(n+1)}{(n+1)^{s}}\right) \\
\leq & A_{\chi}^{c / \sqrt{f_{\chi}}}\left(\sum_{n=1}^{B} \frac{\log ^{2} n}{n}+\int_{B}^{A} \frac{\log ^{2} x}{x} d x\right)+A_{\chi} \frac{\log ^{2}(A+1)}{(A+1)^{s}} \\
\leq & A_{\chi}^{c / \sqrt{f_{\chi}}}\left(\frac{1}{3} \log ^{3} A_{\chi}+c_{B}\right)+A_{\chi} \frac{\log ^{2} A_{\chi}}{A_{\chi}^{s}}
\end{aligned}
$$


where

$$
c_{B}=\sum_{n=1}^{B} \frac{\log ^{2} n}{n}-\frac{1}{3} \log ^{3} B
$$

Hence,

$$
\left|L^{\prime \prime}(s, \chi)\right| \leq A_{\chi}^{c / \sqrt{f_{\chi}}}\left(\frac{1}{3} \log ^{3} A_{\chi}+\log ^{2} A_{\chi}+c_{B}\right)
$$

which yields the desired result (notice that $A_{\chi}^{c / \sqrt{f_{\chi}}}=1+o(1)$ ).

REMARK 19. $B \mapsto c_{B}$ decreases in the range $B \geq 7$ and $c_{B} \leq 0$ for $B \geq 3461$. To obtain the explicit bounds, we choose $B=24$ and in that case $0.200<c_{B}<0.201$.

6.2. Explicit bounds for $L^{\prime}\left(1, \chi_{d}\right)$

Lemma 20. Set

$$
c_{7}=\pi \gamma-\frac{\pi}{2} \log 3+4 \pi \sum_{m \geq 1} \frac{1}{m\left(e^{\pi m \sqrt{3}}-1\right)}=0.092 \ldots
$$

Then $d>4$ implies

$$
L^{\prime}\left(1, \chi_{-d}\right) \leq \frac{\pi^{2}}{6} \sum_{a, b, c} \frac{1}{a}+c_{7} \frac{h(-d)}{\sqrt{d}}
$$

Proof. We have

$$
\lim _{s \rightarrow 1}\left(\zeta(s) L\left(s, \chi_{-d}\right)-\frac{L\left(1, \chi_{-d}\right)}{s-1}\right)=\gamma L\left(1, \chi_{-d}\right)+L^{\prime}\left(1, \chi_{-d}\right) .
$$

Since

$$
\zeta_{K}(s)=\frac{1}{2(\sqrt{d})^{s}} \sum_{a, b, c} \zeta_{Q}(s) \quad(d>4)
$$

and since Dirichlet's class number formula gives

$$
L\left(1, \chi_{-d}\right)=\frac{\pi h(-d)}{\sqrt{d}}=\frac{\pi}{\sqrt{d}} \sum_{a, b, c} 1 \quad(d>4)
$$

we obtain

$$
\text { (30) } \lim _{s \rightarrow 1}\left(\zeta(s) L\left(s, \chi_{-d}\right)-\frac{L\left(1, \chi_{-d}\right)}{s-1}\right)=\frac{1}{2 \sqrt{d}} \sum_{a, b, c} \lim _{s \rightarrow 1}\left(\zeta_{Q}(s)-\frac{2 \pi}{s-1}\right) \text {. }
$$

Now, according to Kronecker's limit formula as given in Selberg [31, (39)], 
we have

$$
\begin{aligned}
\lim _{s \rightarrow 1}\left(\zeta_{Q}(s)-\frac{2 \pi}{s-1}\right)= & \frac{2 \sqrt{d}}{a} \cdot \frac{\pi^{2}}{6}+4 \pi \gamma+2 \pi \log \frac{a}{\sqrt{d}} \\
& +8 \pi \sum_{m \geq 1} \sigma_{-1}(m) \cos \left(\frac{m \pi b}{a}\right) e^{-\pi m \sqrt{d} / a}
\end{aligned}
$$

with

$$
\sigma_{-1}(m)=\sum_{d \mid m} \frac{1}{d}
$$

Now recall that $a \leq \sqrt{d / 3}$. Therefore, we have

$$
\left|\sum_{m \geq 1} \sigma_{-1}(m) \cos \left(\frac{m \pi b}{a}\right) e^{-\pi m \sqrt{d} / a}\right| \leq \sum_{m \geq 1} \sigma_{-1}(m) e^{-\pi m \sqrt{3}} .
$$

Also,

$$
\sum_{m \geq 1} \sigma_{-1}(m) x^{m}=\sum_{m \geq 1} \sum_{d \mid m} \frac{1}{d} x^{m}=\sum_{d \geq 1} \sum_{n \geq 1} \frac{1}{d} x^{d n}=\sum_{d \geq 1} \frac{1}{d\left(1 / x^{d}-1\right)}
$$

and we get

$$
\lim _{s \rightarrow 1}\left(\zeta_{Q}(s)-\frac{2 \pi}{s-1}\right)-\frac{2 \sqrt{d}}{a} \cdot \frac{\pi^{2}}{6} \leq 4 \pi \gamma-\pi \log 3+8 \pi \sum_{m \geq 1} \frac{1}{m\left(e^{\pi m \sqrt{3}}-1\right)} .
$$

We use this in (29) and (30), which completes the proof.

Lemma 21. Let $N \geq 1$ denote a positive integer, and $\omega(a)$ the number of distinct prime divisors of a positive integer $a \geq 1$. Set $\lambda_{1}=0$,

$$
\lambda_{N}=\sum_{a=1}^{N-1} 2^{\omega(a)}\left(\frac{1}{a}-\frac{1}{N}\right)
$$

for $N \geq 2$, and

$$
S(d)=\sum_{a, b, c} \frac{1}{a} .
$$

Then, for any $d>4$,

$$
S(d) \leq \frac{1}{N} h(-d)+\lambda_{N}
$$

In particular,

$$
\begin{array}{llrl}
S(d) & \leq h(-d), & S(d) & \leq \frac{1}{3} h(-d)+1 \\
S(d) & \leq \frac{1}{2} h(-d)+\frac{1}{2}, & S(d) & \leq \frac{1}{4} h(-d)+\frac{17}{12} .
\end{array}
$$

Proof. We use induction on $N \geq 1$. If $N=1$ then we must prove that $S(d) \leq h(-d)$, which is clear. Assume that for some $N \geq 2$ we have proven 
that

$$
S(d) \leq \frac{1}{N-1} h(-d)+\lambda_{N-1} \quad \text { for all } d>4 .
$$

Since for a given $a \geq 1$ there are at most $2^{\omega(a)}$ reduced forms $Q(x, y)=$ $a x^{2}+b x y+c y^{2}$ of discriminant $-d$, we obtain

$$
S(d) \leq \sum_{a=1}^{N-1} \frac{2^{\omega(a)}}{a}+\frac{1}{N}\left(h(-d)-\sum_{a=1}^{N-1} 2^{\omega(a)}\right) \leq \frac{1}{N} h(-d)+\lambda_{N}
$$

for $h(-d) \geq \sum_{a=1}^{N-1} 2^{\omega(a)}$. Suppose now that $h(-d) \leq \sum_{a=1}^{N-1} 2^{\omega(a)}$. Then

$$
\begin{aligned}
\frac{h(-d)}{N(N-1)} \leq & \left(\sum_{a=1}^{N-2} \frac{2^{\omega(a)}}{N(N-1)}\right)+\frac{2^{\omega(N-1)}}{N(N-1)} \\
= & \left(\sum_{a=1}^{N-2} 2^{\omega(a)}\left(\frac{1}{a}-\frac{1}{N}\right)\right)-\left(\sum_{a=1}^{N-2} 2^{\omega(a)}\left(\frac{1}{a}-\frac{1}{N-1}\right)\right) \\
& +2^{\omega(N-1)}\left(\frac{1}{N-1}-\frac{1}{N}\right) \\
= & \lambda_{N}-\lambda_{N-1}
\end{aligned}
$$

and

$$
\frac{1}{N-1} h(-d)+\lambda_{N-1} \leq \frac{1}{N} h(-d)+\lambda_{N}
$$

Since we have assumed that $S(d) \leq \frac{1}{N-1} h(-d)+\lambda_{N-1}$, we deduce that $S(d) \leq \frac{1}{N} h(-d)+\lambda_{N}$, which concludes the proof.

6.3. Proof of Theorem 17. Let $c>0$ be given and set

$$
M_{c}(d)=\max _{1-c / \sqrt{d} \leq s \leq 1}\left|L^{\prime \prime}\left(s, \chi_{-d}\right)\right|
$$

(according to Lemma 18 we have $M_{c}(d) \leq c_{8} \log ^{3} d$ where the constant involved in this bound depends on $c$ only). According to Taylor's formula, for any $s$ in the range $1-c / \sqrt{d} \leq s \leq 1$ we have

$$
L\left(s, \chi_{-d}\right) \geq L\left(1, \chi_{-d}\right)-\frac{c}{\sqrt{d}} L^{\prime}\left(1, \chi_{-d}\right)-\frac{c^{2}}{2 d} M_{c}(d)
$$

and we will have $L\left(s, \chi_{-d}\right)>0$ for all $s$ in the range $1-c / \sqrt{d} \leq s \leq 1$ provided that $d$ is such that

$$
L\left(1, \chi_{-d}\right)>\frac{c}{\sqrt{d}} L^{\prime}\left(1, \chi_{-d}\right)+\frac{c^{2}}{2 d} M_{c}(d)
$$


Since $L\left(1, \chi_{-d}\right)=\pi h(-d) / \sqrt{d}$ for $d>4$ and since according to Lemmas 20 and 21 we have

$$
L^{\prime}\left(1, \chi_{-d}\right) \leq \frac{\pi^{2}}{6} S(d)+c_{7} \frac{h(-d)}{\sqrt{d}} \leq \frac{\pi^{2}}{6}\left(\frac{1}{N} h(-d)+\lambda_{N}\right)+c_{7} \frac{h(-d)}{\sqrt{d}},
$$

we will have $L\left(s, \chi_{-d}\right)>0$ for all $s$ in the range $1-c / \sqrt{d} \leq s \leq 1$ provided that $d$ is such that

$$
1>\frac{\pi c}{6 N}+\frac{\pi c \lambda_{N}}{6 h(-d)}+\frac{c c_{7}}{\pi \sqrt{d}}+\frac{c^{2} M_{c}(d)}{2 \pi h(-d) \sqrt{d}}=\frac{\pi c}{6 N}+o(1)
$$

for we have $\lim _{d \rightarrow \infty} h(-d)=\infty$ (and this can be made effective by using Osterlé's explicit form of Gross-Zagier's bounds for $h(-d)$, see [7]). Now, for a given $c$, if we choose a positive integer $N>6 /(\pi c)$, then we do find that for some effective $D_{c}$ we have $L\left(s, \chi_{-d}\right)>0$ for all $s$ in the range $1-c / \sqrt{d} \leq s \leq 1$ if $d>D_{c}$.

To prove the last assertion of Theorem 17, we use the solutions to the class number 1, 2, 3, 4 and 5 problems (see Goldfeld [7], Arno [1, Theorem 7] and Wagner [34, Table 1]). The following table shows the various constants $D_{c}$ we get for $c=6 / \pi$ depending on which class number problems we assume to be solved. For example, the first line of the table shows that we can take $D_{c}=94704$ if we only use the fact that $h(-d) \geq 2$ for $d>163$, the second line shows that we can take $D_{c}=11357$ if we only use the fact that $h(-d) \geq 3$ for $d>427$, and so forth.

Table 7

\begin{tabular}{cccccc}
\hline$h$ & Last $d$ for which $h(-d)=h$ & $c_{8}$ & $N$ & $\lambda_{N}$ & $D_{c}$ \\
\hline 1 & 163 & 0.173 & 2 & $1 / 2$ & 94704 \\
2 & 427 & 0.219 & 2 & $1 / 2$ & 11357 \\
3 & 907 & 0.283 & 3 & 1 & 2375 \\
4 & 1555 & 0.309 & 3 & 1 & 1556 \\
\hline
\end{tabular}

With these values, it is easy to check that Theorem 17 holds. Indeed, it is easy to perform computer calculations and prove that there are no Siegel zeros for the Dedekind zeta functions of the imaginary quadratic fields of discriminants $-d>-1556$ (see [21, Theorem 5]). This proves the last assertion of the theorem.

7. Proof of the main theorem (Theorem 2). Let $k$ be a totally real number field of degree $n$ and let $K$ be a totally imaginary quadratic extension of $k$. We have $D_{K} \geq D_{k}^{2}$ and then $\sqrt{D_{K} / D_{k}} \geq D_{K}^{1 / 4}$. Furthermore we have $Q_{K} w_{K} \geq 2$, and using both in (1), we get 


$$
h_{K}^{-} \geq 2 \cdot \frac{D_{K}^{1 / 4}}{(2 \pi)^{n}} \cdot \frac{\operatorname{Res} \zeta_{K}}{\operatorname{Res} \zeta_{k}} .
$$

\subsection{Bounds assuming the Generalized Riemann Hypothesis}

7.1.1. Normal case. We use (31) and the bounds on residues we obtained in Theorems 8 and 10 (notice that $E_{\sigma}=1$ under the assumption of the Generalized Riemann Hypothesis) to get, for $D_{K} \geq \varrho^{2 n}$,

$$
h_{K}^{-} \geq 2\left(\frac{C_{5}(n)^{c_{4}(n)}}{2 \pi}\right)^{n} \frac{D_{K}^{1 / 4-c_{4}(n) / 2}}{c_{6}(2 n, \varrho) \log \log D_{K}} .
$$

For the values of $D_{K} \geq \varrho^{2 n}$ we consider, this lower bound is an increasing function of $D_{K}$. Thus we are entitled to use Odlyzko's bounds $D_{K}^{1 /(2 n)} \geq$ $D_{k}^{1 / n} \geq \varrho_{n}$, and we find that $h_{K}^{-}>1$ if $n \geq 83(2 n \geq 166)$, and for degrees $\geq 24$, we get the lower bounds for $D_{K}^{1 /(2 n)}$ as given in Theorem 2 .

For degrees $n \leq 23$, we use the first assertion of Theorem 9 combined with the lower bounds of Corollary 13. We obtain

$$
h_{K}^{-} \geq \frac{1}{2 n c_{6}(2 n, \varrho)}\left(\frac{1}{e \pi}\right)^{n}\left(\frac{\sqrt{\varrho_{K}}}{\log \varrho_{K}}\right)^{n} \frac{\log D_{K}}{\log \log D_{K}} .
$$

Both the functions $\sqrt{\varrho_{K}} / \log \varrho_{K}$ and $\log D_{K} /\left(\log \log D_{K}\right)$ are increasing for $\varrho_{K} \geq \varrho$, and we get the lower bounds for $D_{K}^{1 /(2 n)}$ as given in Theorem 2 .

Also, our bounds can be used to compute a lower bound for the relative class number $h_{K}^{-}$. For example, using the constants for the degree $n=83$ and the Odlyzko bound $\varrho_{K} \geq \varrho_{n}=54.8874$, we find:

Proposition 22. Assume the Generalized Riemann Hypothesis. Then the relative class numbers $h_{K}^{-}$of the normal CM-fields $K$ of degree $2 n \geq 166$ satisfy

$$
h_{K}^{-} \geq \frac{1}{3.2176} \cdot \frac{(1.03937)^{n}}{\log (8.02 n)} .
$$

REMARK. If we used Theorem 14 instead of Corollary 13, we would have a slightly easier proof but the resulting bounds would not be as good as the ones we give.

7.1.2. Non-normal case. We use (31) and the bounds on residues we obtained in Theorems 8 and 14 (again we have $E_{\sigma}=1$ ) to get

$$
h_{K}^{-} \geq 2\left(\frac{C_{5}(n)^{c_{4}(n)}}{2 \pi}\right)^{n} \frac{D_{K}^{1 / 4-c_{4}(n) / 2}}{e^{3 / 2}\left(\log D_{K}\right)^{1 / 2}} \exp \left(-\frac{1}{\left(\log D_{K}\right)^{1 / 2}}\right) .
$$

For the values of $D_{K} \geq \varrho^{2 n}$ we consider, this lower bound is an increasing function of $D_{K}$. Thus we are entitled to use Odlyzko's bounds $D_{K}^{1 /(2 n)} \geq$ $D_{k}^{1 / n} \geq \varrho_{n}$, and we find that $h_{K}^{-}>1$ if $n \geq 88(2 n \geq 176)$, and for degrees 
$\geq 25$, we get the lower bounds for $D_{K}^{1 /(2 n)}$ as given in Theorem 2. This solves conditionally the problem of getting reasonable bounds on degrees of CM-fields with class number one, including those that are not normal.

For degrees $n \leq 24$, we use the first assertion of Theorem 9 combined with the lower bounds of Theorem 14 to obtain

$$
h_{K}^{-} \geq \frac{1}{2 n e^{3 / 2}}\left(\frac{1}{e \pi}\right)^{n}\left(\frac{\sqrt{\varrho_{K}}}{\log \varrho_{K}}\right)^{n} \log D_{K} \exp \left(-\frac{1}{\left(\log D_{K}\right)^{1 / 2}}\right) .
$$

We thus get the lower bounds for $D_{K}^{1 /(2 n)}$ as given in Theorem 2 .

7.2. Unconditional bounds. Using Theorems 8 and 9, Lemma 15, Theorem 16 and Theorem 17, we will now obtain the unconditional lower bound for the relative class number of normal CM-fields.

Assume that $K$ is a normal CM-field of degree $2 n$, with $k$ its maximal totally real subfield, of degree $n$. By Lemma $15, \zeta_{K}(s)$ has at most two zeros counted with multiplicity in the interval $\left[1-1 /\left(\kappa \log D_{K}\right), 1[\right.$, with $\kappa=(2+\sqrt{3}) / 4=0.93301 \ldots$ One of three cases must happen:

1. $\zeta_{k}(s)$ has a zero $\beta \in\left[1-1 /\left(\kappa \log D_{K}\right), 1\left[\right.\right.$. Then $\zeta_{K}(\beta)=0$. Using Theorem 16 and Theorem 8 in formula (31), we get

$$
h_{K}^{-} \geq 2 \cdot \frac{1-\beta}{E_{\sigma}}\left(\frac{C_{5}(n)^{c_{4}(n)}}{2 \pi}\right)^{n} \frac{D_{K}^{1 / 4-c_{4}(n) / 2}}{2 e^{1 /(2 \kappa)}}
$$

where $E_{\sigma}=(1-\beta) /(\sigma-\beta)$ was defined in Lemma 6 , and $\sigma$ is the value we computed in Section 3.4. We see that the term $1-\beta$ in the numerator of this lower bound cancels with the term $1-\beta$ in $E_{\sigma} \leq(1-\beta) /(\sigma-1)$. Thus we get

$$
h_{K}^{-} \geq \frac{\sigma-1}{e^{1 /(2 \kappa)}}\left(\frac{C_{5}(n)^{c_{4}(n)}}{2 \pi}\right)^{n} D_{K}^{1 / 4-c_{4}(n) / 2} .
$$

2. $\zeta_{k}(s)$ has no zero in the range $\left[1-1 /\left(\kappa \log D_{K}\right), 1\left[\right.\right.$, but $\zeta_{K}(s)$ has a simple zero there. Then by [32, Theorem 3] (see also [10]), there exists an imaginary quadratic subfield $F$ of $K$ for which $\zeta_{F}(\beta)=0$. By Theorem 17, we know that

$$
1-\beta>\frac{6}{\pi} \cdot \frac{1}{\sqrt{D_{F}}} \geq \frac{6}{\pi} \cdot \frac{1}{D_{K}^{1 /(2 n)}}
$$

since $D_{F} \leq D_{K}^{1 / n}$.

Using Theorem 16 and Theorem 8 in formula (31) (notice that $E_{\sigma}=1$ here), we get unconditionally

$$
h_{K}^{-} \geq \frac{6}{\pi e^{1 /(2 \kappa)}}\left(\frac{C_{5}(n)^{c_{4}(n)}}{2 \pi}\right)^{n} D_{K}^{1 / 4-c_{4}(n) / 2-1 /(2 n)} .
$$

3. $\zeta_{k}(s)$ has no zero in the range $\left[1-1 /\left(\kappa \log D_{K}\right), 1\left[\right.\right.$ and $\zeta_{K}(s)$ has no simple zero there. In this case, either $\zeta_{K}$ has no zero at all there, or has a 
double zero there, and in both cases we have $\zeta_{K}\left(1-1 /\left(\kappa \log D_{K}\right)\right) \leq 0$, so that

$$
h_{K}^{-} \geq \frac{2}{\kappa e^{1 /(2 \kappa)}}\left(\frac{C_{5}(n)^{c_{4}(n)}}{2 \pi}\right)^{n} \frac{D_{K}^{1 / 4-c_{4}(n) / 2}}{\log D_{K}} .
$$

Now that we have taken care of all the three possible cases, we work in two ways.

- If we do not want to obtain lower bounds for the relative class number $h_{K}^{-}$increasing to infinity with the degree $2 n$ of $K$ but only want an upper bound for the degrees $2 n$ of the CM-fields $K$ of relative class number one, we can immediately eliminate case 2 . Indeed, by [25], if $F$ is an imaginary quadratic subfield of a CM-field $K$ with relative class number $h_{K}^{-}$equal to one, then $h_{F}$ divides 4 , thus $h_{F}=1,2$ or 4 and $D_{F} \leq 1555$ (see Section 6.3). But we know that the zeta functions $\zeta_{F}$ of such fields $F$ have no real zeros in the range $0<s<1$. Only cases 1 and 3 remain, and it is enough to check that both lower bounds (32) and (34) imply $h_{K}^{-}>1$ for $D_{K} \geq \varrho^{2 n}$ (as in the previous sections, we check that those lower bounds in cases 1 and 3 are increasing functions of $D_{K}$ for $\left.D_{K} \geq \varrho^{2 n}\right)$. Using L. Tartar's bounds $D_{K}^{1 /(2 n)} \geq D_{k}^{1 / n} \geq \varrho_{n}^{\prime}$, we find that $h_{K}^{-}>1$ if $n \geq 134$ (that is, $2 n \geq 268$ ), and for degrees $n \geq 27$ we get the lower bounds for $D_{K}^{1 /(2 n)}$ as given in Theorem 2.

- If we do want to obtain lower bounds for the relative class number $h_{K}^{-}$ increasing to infinity with the degree $2 n$ of $K$ (see for example Proposition 23 below), we must check that each of the three lower bounds (32)-(34) implies that $h_{K}^{-}>1$ for $D_{K} \geq \varrho^{2 n}$.

Proposition 23. If we do not assume the Generalized Riemann Hypothesis, then the relative class numbers $h_{K}^{-}$of the normal CM-fields $K$ of degree $2 n \geq 268$ satisfy

$$
h_{K}^{-} \geq \frac{(1.0515)^{n}}{6.057 n} .
$$

Proof. We use the constants for $n=134$ and Tartar's bound $\varrho_{K} \geq \varrho_{n}^{\prime}=$ 44.6377 (see [28]). For this lower bound of the root discriminant, we see that (34) is the worst of the three lower bounds, and we get the desired result.

For degrees $n \leq 26$, we use Theorem 9 instead of Theorem 8 and proceed as above. By Lemma $15, \zeta_{K}(s)$ has at most two zeros counted with multiplicity in the interval $\left[1-1 /\left(\kappa \log D_{K}\right), 1[\right.$. One of three cases can happen:

1. $\zeta_{k}$ has a zero $\beta \in\left[1-1 /\left(\kappa \log D_{K}\right), 1\left[\right.\right.$. Then $\zeta_{K}(\beta) \leq 0$. We use the second assertion of Theorem 9 , the $1-\beta$ terms cancel each other and we obtain

$$
h_{K}^{-} \geq \frac{2}{(e \pi)^{n} e^{1 /(2 \kappa)}}\left(\frac{\sqrt{\varrho_{K}}}{\log \varrho_{K}}\right)^{n} .
$$


2. $\zeta_{k}$ has no zero in the range $\left[1-1 /\left(\kappa \log D_{K}\right), 1\left[\right.\right.$, but $\zeta_{K}$ has a simple zero there. Then by [32, Theorem 3], there exists an imaginary quadratic subfield $F$ of $K$ for which $\zeta_{F}(\beta)=0$. By Theorem 17, and since $h_{F}$ divides 4 if $h_{K}^{-}=1$, we obtain $D_{F} \leq 1555$, and we know that those imaginary quadratic number fields have no Siegel zeros.

3. $\zeta_{k}$ has no zero in the range $\left[1-1 /\left(\kappa \log D_{K}\right), 1\left[\right.\right.$ and $\zeta_{K}$ has no simple zero there. In this case, whether or not there is a double zero, we obtain

$$
h_{K}^{-} \geq \frac{1}{2 n(e \pi)^{n} \kappa e^{1 /(2 \kappa)}}\left(\frac{\sqrt{\varrho_{K}}}{\log \varrho_{K}}\right)^{n} .
$$

We check that this last case is the worst numerically and we obtain the lower bounds for $D_{K}^{1 /(2 n)}$ as given in Theorem 2 for degrees $\leq 26$.

Acknowledgements. It is a great pleasure for us to thank our Ph.D. advisor, S. Louboutin, for his help while writing this paper and for having presented us with the issue dealt with in this paper. We also thank J. Oesterlé who kindly sent us Theorem 14 and its proof.

Appendix. We give here the verbatim proof of Theorem 14 as sent to us by J. Oesterlé.

Soit $k$ un corps de nombres. Notons $n$ son degré, $r_{1}$ le nombre de ses places réelles, $r_{2}$ le nombre de ses places complexes, $d$ la valeur absolue de son discriminant, $\zeta_{k}$ sa fonction zêta de Dedekind et $\kappa$ le résidu en 1 de $\zeta_{k}$.

ThÉORÈme 1. Si $\zeta_{k}$ satisfait l'hypothèse de Riemann et $k \neq \mathbb{Q}$, on a

$$
\kappa \geq \frac{e^{-3 / 2}}{\sqrt{\log d}} \exp \left(\frac{-1}{\sqrt{\log d}}\right) .
$$

Posons $\xi_{k}(s)=s(s-1) d^{s / 2} \Gamma_{R}(s)^{r_{1}+r_{2}} \Gamma_{R}(s+1)^{r_{2}} \zeta_{k}(s)$, avec $\Gamma_{R}(s)=$ $\pi^{-s / 2} \Gamma(s / 2)$. La fonction $\xi_{k}$ se prolonge en une fonction entière d'ordre 1 , qui satisfait l'équation fonctionnelle $\xi_{k}(1-s)=\xi_{k}(s)$. Nous admettons dans la suite que $\zeta_{k}$ satisfait l'hypothèse de Riemann, i.e. que tous les zéros de $\xi_{k}$ ont pour partie réelle $1 / 2$. La fonction $\xi_{k}$ possède le développement en produit de Weierstraß

$$
\xi_{k}(s)=\xi_{k}(0) \prod_{\varrho}^{*}\left(1-\frac{s}{\varrho}\right),
$$

où le produit est indexé par les zéros $\varrho$ de $\xi_{k}$ (répétés un nombre de fois égal à leur multiplicité) et où $\prod^{*}$ signifie que l'on effectue le produit après avoir regroupé les termes correspondant à $\varrho$ et $1-\varrho$ (pour en assurer la convergence normale sur tout compact de $\mathbb{C}$ ). On en déduit, avec des conventions 
analogues sur les sommes,

$$
\frac{\xi_{k}^{\prime}}{\xi_{k}}(s)=\sum_{\varrho}^{*} \frac{1}{s-\varrho} .
$$

Posons $\varphi(s)=s \Gamma_{R}(s)^{r_{1}+r_{2}} \Gamma_{R}(s+1)^{r_{2}}$, de sorte que

$$
\xi_{k}(s)=(s-1) d^{s / 2} \varphi(s) \zeta_{k}(s) \text {. }
$$

Lemme 1. La fonction $\varphi^{\prime} / \varphi$ est croissante sur $] 0,+\infty[$. La fonction $f$ : $x \mapsto x \frac{\varphi^{\prime}}{\varphi}(x)$ est convexe sur $] 0,+\infty[$; elle est majorée par $f(1)$ sur $[1,2]$.

On a $\varphi(s)=\varphi_{0}(s) \varphi_{1}(s)^{r_{1}+r_{2}-1} \varphi_{2}(s)^{r_{2}}$, où $\varphi_{1}(s)=\Gamma_{R}(s), \varphi_{2}(s)=$ $\Gamma_{R}(s+1), \varphi_{0}(s)=s \Gamma_{R}(s)=2 \pi \Gamma_{R}(s+2)$. Il suffit de démontrer le lemme séparément pour chacune des fonctions $\varphi_{i}$. La fonction $\Gamma$ est logarithmiquement convexe sur $] 0,+\infty\left[\right.$. Il en est donc de même de $\Gamma_{R}$, ce qui implique que chacune des fonctions $\varphi_{i}^{\prime} / \varphi_{i}$ est croissante sur $] 0,+\infty[$.

Posons $f_{i}(x)=x \frac{\varphi_{i}^{\prime}}{\varphi_{i}}(x)$. Comme

$$
\frac{\Gamma^{\prime}}{\Gamma}(x)=-\gamma-\frac{1}{x}+\sum_{n=1}^{\infty}\left(\frac{1}{n}-\frac{1}{x+n}\right) \text {, }
$$

on a

$$
\begin{aligned}
& f_{1}(x)=-\frac{x}{2}(\log \pi+\gamma)-1+\sum_{n=1}^{\infty}\left(\frac{x}{2 n}-\frac{x}{x+2 n}\right) \\
& f_{2}(x)=-\frac{x}{2}(\log \pi+\gamma)-\frac{x}{x+1}+\sum_{n=1}^{\infty}\left(\frac{x}{2 n}-\frac{x}{x+2 n+1}\right)
\end{aligned}
$$

et $f_{0}(x)=1+f_{1}(x)$, de sorte que les dérivées secondes

$$
f_{0}^{\prime \prime}(x)=f_{1}^{\prime \prime}(x)=2 \sum_{n=1}^{\infty} \frac{2 n}{(x+2 n)^{3}} \quad \text { et } \quad f_{2}^{\prime \prime}(x)=2 \sum_{n=0}^{\infty} \frac{2 n+1}{(x+2 n+1)^{3}}
$$

sont positives sur $] 0,+\infty\left[\right.$ et que les fonctions $f_{i}$ sont convexes sur $] 0,+\infty[$.

Pour démontrer que $f_{i}$ est majorée par $f_{i}(1)$ sur [1,2], il suffit donc de vérifier que l'on a $f_{i}(2) \leq f_{i}(1)$, ce qui résulte des égalités $f_{1}(1)=$ $-\frac{1}{2}(\log \pi+\gamma)-\log 2, f_{1}(2)=-(\log \pi+\gamma), f_{2}(1)=-\frac{1}{2}(\log \pi+\gamma), f_{1}(2)=$ $-(\log \pi+\gamma)+2-2 \log 2, f_{0}(1)=f_{1}(1)+1$ et $f_{0}(2)=f_{1}(2)+1$.

Lemme 2. Soit c un nombre réel $>0$. On a

$$
\frac{\xi_{k}(1+c)}{\xi_{k}(1)} \geq \frac{c}{\kappa} d^{c / 2} \exp \left(c \frac{\varphi^{\prime}}{\varphi}(1)\right) .
$$

On a $\zeta_{k}(1+c) \geq 1$ et $\varphi^{\prime} / \varphi$ est croissante sur $] 0,+\infty[$ d'après le lemme 1 , d'où

$$
\frac{\xi_{k}(1+c)}{\xi_{k}(1)}=\frac{c d^{c / 2} \varphi(1+c) \zeta_{k}(1+c)}{\kappa \varphi(1)} \geq \frac{c d^{c / 2} \varphi(1+c)}{\kappa \varphi(1)} \geq \frac{c}{\kappa} d^{c / 2} \exp \left(c \frac{\varphi^{\prime}}{\varphi}(1)\right) .
$$


Lemme 3. Soit c un nombre réel $>0$. On a

$$
\frac{\xi_{k}(1+c)}{\xi_{k}(1)} \leq d^{\left(c+c^{2}\right) / 2} \exp \left(1+c+c(1+c) \frac{\varphi^{\prime}}{\varphi}(1+c)\right) .
$$

On a pour $0 \leq t \leq c$ et pour tout zéro $\varrho$ de $\xi_{k}$ (de partie réelle $1 / 2$ par hypothèse),

$$
\begin{aligned}
\operatorname{Re}\left(\frac{1}{1+t-\varrho}\right) & \leq \frac{1+2 c}{1+2 t} \operatorname{Re}\left(\frac{1}{1+c-\varrho}\right) \\
& \leq(1+2(c-t)) \operatorname{Re}\left(\frac{1}{1+c-\varrho}\right)
\end{aligned}
$$

d'où, en sommant sur $\varrho$,

$$
\frac{\xi_{k}^{\prime}}{\xi_{k}}(1+t) \leq(1+2(c-t)) \frac{\xi_{k}^{\prime}}{\xi_{k}}(1+c)
$$

et en intégrant

$$
\frac{\xi_{k}(1+c)}{\xi_{k}(1)}=\exp \left(\int_{0}^{c} \frac{\xi_{k}^{\prime}}{\xi_{k}}(1+t) d t\right) \leq \exp \left(\left(c+c^{2}\right) \frac{\xi_{k}^{\prime}}{\xi_{k}}(1+c)\right) .
$$

On a

$$
\frac{\xi_{k}^{\prime}}{\xi_{k}}(1+c)=\frac{1}{c}+\frac{1}{2} \log d+\frac{\varphi^{\prime}}{\varphi}(1+c)+\frac{\zeta_{k}^{\prime}}{\zeta_{k}}(1+c)
$$

et

$$
\frac{\zeta_{k}^{\prime}}{\zeta_{k}}(1+c)=-\sum_{\mathfrak{p}} \frac{(\mathrm{N}(\mathfrak{p}))^{-(1+c)}}{1-(\mathrm{N}(\mathfrak{p}))^{-(1+c)}} \log \mathrm{N}(\mathfrak{p}) \leq 0
$$

(où $\mathfrak{p}$ parcourt l'ensemble des idéaux maximaux de l'anneau des entiers de $K$ et $\mathrm{N}(\mathfrak{p})$ désigne la norme de $\mathfrak{p})$. Le lemme 3 résulte de ces trois dernières relations.

Lemme 4. Pour tout nombre réel $c \in] 0,1]$, on a

$$
\kappa \geq c \exp \left(-1-c-\frac{c^{2}}{2} \log d\right)
$$

En combinant les lemmes 2 et 3 , on obtient

$$
\kappa \geq c d^{-c^{2} / 2} \exp \left(-1-c+c \frac{\varphi^{\prime}}{\varphi}(1)-c(1+c) \frac{\varphi^{\prime}}{\varphi}(1+c)\right) .
$$

Par ailleurs, si l'on pose $f(x)=x \frac{\varphi^{\prime}}{\varphi}(x)$, on a $f(1) \geq f(1+c)$ d'après le lemme 1 , c'est-à-dire $\frac{\varphi^{\prime}}{\varphi}(1)-(1+c) \frac{\varphi^{\prime}}{\varphi}(1+c) \geq 0$.

Le théorème 1 se déduit du lemme 4 en prenant $c=1 / \sqrt{\log d}$, ce qui est légitime $\operatorname{si} \log d \geq 1$, i.e. $k \neq \mathbb{Q}$. 


\section{References}

[1] S. Arno, The imaginary quadratic fields of class number 4, Acta Arith. 60 (1992), $321-334$.

[2] S. Bessassi, Bounds for the degrees of the CM-fields of class number one, Ph.D. thesis, Univ. de Caen, 2001.

[3] R. P. Boas and M. Kac, Inequalities for Fourier transforms of positive functions, Duke Math. J. 12 (1945), 189-206.

[4] J. Buchmann and H. C. Williams, On the computation of the class number of an algebraic number field, Math. Comp. 53 (1989), 679-688.

[5] K.-Y. Chang and S.-H. Kwon, The non-abelian normal CM-fields of degree 36 with class number one, Acta Arith. 101 (2002), 53-61.

[6] G. Cornell and L. C. Washington, Class numbers of cyclotomic fields, J. Number Theory 21 (1985), 260-274.

[7] D. Goldfeld, Gauss' class number problem for imaginary quadratic fields, Bull. Amer. Math. Soc. 13 (1985), 23-37.

[8] - An asymptotic formula relating the Siegel zero and the class number of quadratic fields, Ann. Scuola Norm. Sup. Pisa (4) 2 (1975), 611-615.

[9] D. Goldfeld and A. Schinzel, On Siegel's zero, ibid., 571-583.

[10] H. Heilbronn, On real zeros of Dedekind zeta-functions, Canad. J. Math. 25 (1973), 870-873.

[11] J. Hoffstein, Some analytic bounds for zeta functions and class numbers, Invent. Math. 55 (1979), 37-47.

[12] Y. Lefeuvre, Corps diédraux à multiplication complexe principaux, Ann. Inst. Fourier (Grenoble) 50 (2000), 67-103.

[13] Y. Lefeuvre and S. Louboutin, The class number one problem for the dihedral CMfields, in: Algebraic Number Theory and Diophantine Equation (Graz, 1998), de Gruyter, Berlin, 2000, 249-275.

[14] F. Lemmermeyer, S. Louboutin and R. Okazaki, The class number one problem for some non-abelian normal CM-fields of degree 24, J. Théor. Nombres Bordeaux 11 (1999), 387-406.

[15] S. Louboutin, The class number one problem for the non-abelian normal CM-fields of degree 16, Acta Arith. 82 (1997), 173-196.

[16] - , The class number one problem for the dihedral and dicyclic CM-fields, Colloq. Math. 80 (1999), 259-265.

[17] - Explicit bounds for residues of Dedekind zeta functions, values of $L$-functions at $s=1$ and relative class numbers, J. Number Theory 85 (2000), 263-282.

[18] -, Explicit upper bounds for residues of Dedekind zeta functions and values of $L$ functions at $s=1$, and explicit lower bounds for relative class numbers of CM-fields, Canad. J. Math. 53 (2001), 1194-1222.

[19] - Explicit lower bounds for residues at $s=1$ of Dedekind zeta functions and relative class numbers of $C M$-fields, preprint.

[20] S. Louboutin and R. Okazaki, The class number one problem for some non-abelian normal CM-fields of 2-power degrees, Proc. London Math. Soc. (3) 76 (1998), 523548.

[21] M. E. Low, Real zeros of the Dedekind zeta function of an imaginary quadratic field, Acta Arith. 14 (1967/68), 117-140.

[22] A. M. Odlyzko, Some analytic estimates of class numbers and discriminants, Invent. Math. 29 (1975), 275-286. 
[23] A. M. Odlyzko, Bounds for discriminants and related estimates for class numbers, regulators and zeros of zeta functions: a survey of recent results, Sém. Théor. Nombres Bordeaux 2 (1990), 119-141.

[24] J. Oesterlé, Versions effectives du théorème de Chebotarev sous l'hypothèse de Riemann généralisée, Astérisque 61 (1979), 165-167.

[25] R. Okazaki, Inclusion of CM-fields and divisibility of relative class numbers, Acta Arith. 92 (2000), 319-338.

[26] Y.-H. Park, The class number one problem for the non-abelian normal CM-fields of degree 24 and 40, ibid. 101 (2002), 63-81.

[27] J. Pintz, Elementary methods in the theory of L-functions II, ibid. 31 (1976), 273289.

[28] G. Poitou, Sur les petits discriminants, Sém. Delange-Pisot-Poitou, 18e année, 1976/77.

[29] —, Minorations de discriminants, Sém. Bourbaki, 28e année, 1975/76, 136-153.

[30] J. B. Rosser and L. Schoenfeld, Approximate formulas for some functions of prime numbers, Illinois J. Math. 6 (1962), 64-94.

[31] A. Selberg and S. Chowla, On Epstein's zeta-function, J. Reine Angew. Math. 227 (1967), 86-110.

[32] H. M. Stark, Some effective cases of the Brauer-Siegel theorem, Invent. Math. 23 (1974), 135-152.

[33] - The analytic theory of algebraic numbers, Bull. Amer. Math. Soc. 81 (1975), 961-972.

[34] C. Wagner, Class number 5, 6 and 7, Math. Comp. 65 (1996), 785-800.

[35] L. C. Washington, Introduction to Cyclotomic Fields, 2nd ed., Grad. Texts in Math. 83, Springer, 1997.

[36] K. Yamamura, The determination of the imaginary abelian number fields with class number one, Math. Comp. 62 (1994), 899-921.

Département de Mathématiques

Université de Caen

Campus 2

Boulevard Maréchal Juin

BP 5186

14032 Caen Cedex, France

E-mail: bessassi@math.unicaen.fr

Received on 19.3.2001

and in revised form on 25.2.2002 